\title{
Robust measures of skewness and kurtosis for macroeconomic and financial time series
}

\author{
Andrea Bastianin \\ University of Milan-Bicocca
}

June 12, 2019

\begin{abstract}
The sample skewness and kurtosis of macroeconomic and financial time series are routinely scrutinized in the early stages of model-building and are often the central topic of studies in economics and finance. Notwithstanding the availability of several robust estimators, most scholars in economics rely on method-of-moments estimation that is known to be very sensitive to outliers. We carry out an extensive Monte Carlo analysis to evaluate the bias and root mean squared error of twelve different estimators of skewness and kurtosis. We consider nine statistical distributions that approximate the range of data generating processes of many macroeconomic and financial time series. Both in independently and identically distributed samples and in data generating processes featuring serial correlation L-moments and trimmed L-moments estimators are particularly resistant to outliers and deliver improvements over standard as well as alternative robust estimators of skewness and kurtosis. The application to 128 macroeconomic and financial time series sourced from a large, monthly frequency, database (i.e. the FRED-MD of McCracken and $\mathrm{Ng}, 2016$ ) confirms the findings of the simulation study.
\end{abstract}

Key Words: FRED-MD; kurtosis; L-moments; outlier; robust statistics; skewness.

JEL Codes: C22, C46, C52, C55, C58.

MSC Codes: 62F35, 62J20, 62M10, 62P20, 91B84, 91G70.

Corresponding author: Andrea Bastianin, Department of Economics, Management, and Statistics, University of Milan - Bicocca, Via Bicocca degli Arcimboldi, 8, Building U7, 20126 - Milan - Italy. Email: andrea. bastianin@unimib.it. 


\section{Introduction}

The assumption of normality is central in many theoretical models and empirical applications in macroeconomics and finance (see e.g. Cecchetti et al., 1990; DeLong and Summers, 1986; Scott and Horvath, 1980). Normality tests, sample skewness and kurtosis of time series are routinely used as diagnostic tools for econometric model-building and to investigate substantive questions in economics and finance. Bontemps and Meddahi (2005) and Kilian and Demiroglu (2000) provide several examples where normality testing is of interest to investigate key issues in economics and finance. While robust estimators and tests do exist, most scholars in economics rely on the method-of-moments to estimate the skewness and kurtosis of data and on the Jarque-Bera test as a diagnostic check. It is well known that measures of skewness and kurtosis based on sample moments are highly sensitive to outliers ${ }^{1}$ and even a single large observation can tear them apart (see e.g. Bonato, 2011; Kim and White, 2004; Brys et al., 2004; Thomas, 2009).

We rely on a Monte Carlo exercise to assess the bias and root mean squared error (RMSE) of twelve estimators of skewness and kurtosis. These include method-of-moments estimators, as well as more robust measures based on quantiles, L-moments and trimmed Lmoments (TL-moments). While the statistical literature on robust estimation is widespread, L-moments are relatively new in time series econometrics. ${ }^{2}$ Much like conventional moments, L-moments can be used to characterize the location, scale and shape of a probability distribution (Hosking, 1990). We show that, both in independently and identically distributed (iid) samples and in data generating processes featuring serial correlation, L-moments and TL-moments estimators are particularly resistant to outliers and deliver improvements over standard as well as other robust measures of skewness and kurtosis. The design based on iid

\footnotetext{
${ }^{1}$ Outliers, are also known as aberrant or influential observations because dropping them changes the estimates by a "large amount" (see e.g. Wooldridge, 2012). They can arise either as a result of measurement errors (e.g. a mistake made when entering the data) or because they genuinely belongs to a data generating process that produces extreme values (e.g. a mixture of two Normal distributions, with one distribution having a much smaller mean than the other and a very small probability to draw from it). In this paper we focus on outliers interpreted as observations that belong to the time series and are not due to measurement errors. See Franses and Van Dijk (2000) and Denby and Martin (1979) for further details of outliers in time series models.

${ }^{2}$ An exception is Darolles et al. (2009) who constructed measures of fund performance based on L-moments to overcome the drawbacks of more traditional performance metrics such as the Sharpe ratio.
} 
samples can be seen as an ideal benchmark when regression residuals are analyzed. The empirical application, that involves 128 macroeconomic and financial time series sourced from a large, monthly frequency, database (i.e. the FRED-MD; see McCracken and Ng, 2016, for details), confirms that L- and TL-moments estimators are particularly resistant to outliers.

Five studies are closely related to this paper. Kim and White (2004) and Bonato (2011) deal with the estimation of skewness and kurtosis in the presence of outlying observations. Although these authors do consider robust measures of skewness and kurtosis, they do not analyze L-, nor TL-moments. Karvanen (2006) proposed estimating quantile mixtures via L-moments and presented an empirical analysis involving stock market returns, however the study did not explicitly focus on the comparative performance of skewness and kurtosis estimators. Thomas (2009) assessed the small sample behavior of measures symmetry based on quantiles, L-moments, and TL-moments with a Monte Carlo analysis, but did not consider measures of kurtosis. Harri and Coble (2011) introduced Normality tests based on L-moments, but focused exclusively on iid samples, while we consider also simulated data from autoregressive processes.

This paper makes three main contributions to this strand of the literature. First, by considering Monte Carlo designs with serially dependent observations, we introduce L- and TL-moments in the time series econometrics literature. Second, we carry out an extensive set of simulations with sample size and outlying observations aimed at mimicking the dataset typically analyzed in macroeconomics and finance. Third, we present a detailed empirical application involving a prominent large, monthly, dataset of macroeconomic and financial time series.

The rest of the paper is organized as follows. Section 2 reviews different estimators of skewness and kurtosis. Sections 3 and 4 present the Monte Carlo and empirical analysis, respectively. Section 5 concludes. An Appendix with additional methodological details and results completes the paper. 


\section{Measures of skewness \& kurtosis}

\section{$2.1 \quad$ Notation}

Let $\left\{X_{t}\right\}_{t=1}^{T}$ be a series with mean $\mu$ and $r$-th central moment $\mu_{r}=E(X-\mu)^{r}$, where $E(\cdot)$ denotes the expectation operator. The second central moment, $\mu_{2}$, is variance of $X_{t}$ and is denoted as $\sigma^{2}$, while its standard deviation is $\sigma$. Moreover, $Q_{\alpha}=F^{-1}(\alpha)$ is the quantile function and $F^{-1}(\cdot)$ is the inverse cumulative distribution function and $\alpha$ is a probability. Lastly, $X_{1: T} \leq X_{2: T} \leq \ldots \leq X_{n: T}$ are the order statistics of a random sample of size $T$ drawn from the distribution of $X_{t}{ }^{3}$

Measures of skewness and kurtosis are denoted as $S K_{j}$ and $K R_{j}^{\prime}$. We focus on excess kurtosis $\left(K R_{j}\right)$, defined as the difference between the $j$-th measure of kurtosis $\left(K R_{j}^{\prime}\right)$ and the corresponding reference value for the Normal distribution. These reference values are shown in the first column of Table $1(b)$. Details on the estimation procedures, as well as further methodological issues are discussed in Sections A and B of the Appendix.

\subsection{Conventional measures of skewness \& kurtosis}

The two most widely used measures of skewness and kurtosis are based on standardized moments. The moment-based skewness is defined as:

$$
S K_{1}=\frac{\mu_{3}}{\sigma^{3}}
$$

for the Normal distribution $S K_{1}=0$. Symmetric distributions have zero skewness, while for asymmetric distributions with longer left (right) tail skewness $S K_{1}$ is negative (positive). ${ }^{4}$

The kurtosis of a distribution measures its tail behavior, that is its propensity to generate observations far away from the center of its support (Westfall, 2014). Sample kurtosis is

\footnotetext{
${ }^{3}$ The $k$-th order statistics of a sample of size $T$ is equal to its $k$-th smallest value. For the sample $\{5,3,9,1\}$, we have: $X_{1: 4}=1, X_{2: 4}=3, X_{3: 4}=5, X_{4: 4}=9$.

${ }^{4}$ Zero skewness however is only necessary, but not sufficient for the symmetry of a distribution (von Hippel, 2010). A case in point is the discrete Student's $t$ distribution (Ord, 1968). In the case of the Normal mixture distribution Meijer (2000) showed that a mixture of two Normal distributions with $\mu_{1}=$ $-2, \sigma_{1}=1, \mu_{2}=1, \sigma_{2}=\sqrt{2}$ and probability of drawing from the first distribution equal to $1 / 3$ has zero central moment but is asymmetric, that is: $E\left(X_{t}-\mu\right) \neq-E\left(X_{t}-\mu\right)$. A third example of an asymmetric distribution with zero skewness is reported by Bai and $\mathrm{Ng}$ (2001, p. 230).
} 
conventionally based on method-of-moments estimation of:

$$
K R_{1}^{\prime}=\frac{\mu_{4}}{\sigma^{4}}
$$

for the Normal distribution $K R_{1}^{\prime}=3$, therefore the excess kurtosis of a distribution is defined as $K R_{1}=K R_{1}^{\prime}-3$. A distribution is thus said to have "fat-tails" if $K R_{1}>3$.

It is well known that both $S K_{1}$ and $K R_{1}$ have a very low breakdown value, in fact a single outlier can severely affect them, leading to extremely large values (Kim and White, 2004; Hubert and Debruyne, 2009). Another pitfall of these measures is that they cannot be defined for distributions that do not posses finite moments up to the fourth order. A case in point is Stable distribution family, often used to describe financial time series (Bonato, 2011; Mittnik and Rachev, 1993; Loretan and Phillips, 1994). Method-of-moments estimation of $S K_{1}$ and $K R_{1}$ is discussed in Section B.1 of the Appendix.

\subsection{Robust measures of skewness}

Given the drawbacks of $S K_{1}$ and $K R_{1}$, both Kim and White (2004) and Bonato (2011) have highlighted the advantages of alternative measures of skewness and kurtosis in financial applications. ${ }^{5}$ Several robust estimators of skewness rely on quantiles and are encompassed by the measure proposed by Hinkley (1975):

$$
S K(\alpha)=\frac{Q_{1-\alpha}+Q_{\alpha}-2 Q_{0.5}}{Q_{1-\alpha}-Q_{\alpha}} \quad \text { for } 0 \leq \alpha \leq 0.5
$$

The quartile skewness due to Bowley (1920) is obtained setting $\alpha=0.25$ :

$$
\begin{aligned}
S K_{2} & =\frac{Q_{0.75}+Q_{0.25}-2 Q_{0.50}}{Q_{0.75}-Q_{0.25}} \\
& =\frac{\left(Q_{0.75}-Q_{0.50}\right)-\left(Q_{0.50}-Q_{0.25}\right)}{Q_{0.75}-Q_{0.25}}
\end{aligned}
$$

where $-1 \leq S K_{2} \leq 1$. The denominator in Equation (4) is the interquartile range and it re-scales the numerator so that $S K_{2}=1\left(S K_{2}=-1\right)$ in the case of extreme right (left)

\footnotetext{
${ }^{5}$ There is a voluminous literature on robust statistics and on tests of symmetry. See e.g. Brys et al. $(2003,2004)$ and references therein.
} 
skewness. For symmetric distributions $\left(Q_{0.75}-Q_{0.50}\right)=\left(Q_{0.50}-Q_{0.25}\right)$ and hence $S K_{2}=0$.

One drawback of the Hinkley's measure of skewness is its dependence on the level of $\alpha$. To overcome this limitation Groeneveld and Meeden (1984) proposed to integrate out $\alpha$ :

$$
S K_{3}=\frac{\int_{0}^{0.5}\left[Q_{1-\alpha}+Q_{\alpha}-2 Q_{0.5}\right] \mathrm{d} \alpha}{\int_{0}^{0.5}\left[Q_{1-\alpha}-Q_{\alpha}\right] \mathrm{d} \alpha}=\frac{\mu-Q_{0.5}}{E\left(\left|X_{t}-Q_{0.5}\right|\right)}
$$

notice that also in this case $-1 \leq S K_{3} \leq 1$ and $S K_{3}=0$ for symmetric distributions.

The denominator of Equation (5) is the mean absolute deviation (MAD); substituting the MAD with the standard deviation, we get the Pearson coefficient of skewness:

$$
S K_{4}=\frac{\mu-Q_{0.5}}{\sigma}
$$

Notice that, unlike $S K_{2}$ and $S K_{3}, S K_{4}$ is not bounded in the $(-1,1)$ interval.

Of the four measures of skewness introduced so far only $S K_{2}$ can be applied to any distribution, independently of whether its moments exist. In fact, $S K_{1}, S K_{3}$ and $S K_{4}$ require the existence of moments up to order three, one and two, respectively.

\subsection{Robust measures of kurtosis}

Moors (1988) proposed to interpret kurtosis of $X_{t}$ as dispersion around $\mu \pm \sigma$ :

$$
K R_{2}^{\prime}=\frac{\left(Q_{3 / 8}-Q_{1 / 8}\right)+\left(Q_{7 / 8}-Q_{5 / 8}\right)}{Q_{6 / 8}-Q_{2 / 8}}
$$

$K R_{2}^{\prime}$ relies on "octiles", that is $Q_{i / 8}=F^{-1}(i / 8)$ for $i=1, \ldots, 7$. The two terms in the numerator are large (small) if relatively little (much) probability mass is concentrated around $Q_{2 / 8}$ and $Q_{6 / 8}$, corresponding with large (small) dispersion in the neighborhoods of $\mu \pm \sigma$. Table $1(b)$ shows that for the standard Normal distribution $K R_{2}^{\prime}=1.233$, therefore the centered measure is: $K R_{2}=K R_{2}^{\prime}-1.233$.

Hogg $(1972,1974)$ introduced a measure of kurtosis based on averages of lower and upper quantiles, defined as $L_{\alpha}=(1 / \alpha) \int_{0}^{\alpha} Q(u) \mathrm{d} u$ and $U_{\alpha}=(1 / \alpha) \int_{1-\alpha}^{1} Q(u) \mathrm{d} u$, respectively. In 
practice, we rely on:

$$
K R_{3}^{\prime}=\frac{U_{0.05}-L_{0.05}}{U_{0.50}-L_{0.50}}
$$

for the standard Normal distribution $K R_{3}^{\prime}=2.585$, therefore: $K R_{3}=K R_{3}^{\prime}-2.585$.

A third measure of kurtosis is due to Crow and Siddiqui (1967):

$$
K R_{4}^{\prime}=\frac{Q_{0.975}+Q_{0.025}}{Q_{0.75}-Q_{0.25}}
$$

as shown in Table $1(b)$ for the standard Normal distribution $K R_{4}^{\prime}=2.906$ and hence $K R_{4}=$ $K R_{4}^{\prime}-2.906$.

Since $K R_{2}^{\prime}$ and $K R_{4}^{\prime}$ depend only on quantiles, they are defined for any distribution, independently on whether they have finite moments or not.

\subsection{Robust measures based on L- and trimmed L-moments}

Hosking (1990) proposed to estimate the location, scale and shape of probability distributions with linear combinations of order statistics. These functions are called "L-moments", where the "L" emphasizes their linearity. Being linear functions of the data L-moments are more robust to outliers than conventional moments that raise the difference from the mean to the third or fourth power. Moreover, since L-moments exist for any random variable with finite mean, they uniquely characterize a class of distributions that is wider than the set of distributions for which conventional moments can be applied. In fact, a distribution with finite mean is uniquely characterized by its L-moments, even when conventional moments do not exist. 
The first four L-moments of a random variable are: ${ }^{6}$

$$
\begin{aligned}
& \lambda_{1}=E\left(X_{1: 1}\right)=\int_{0}^{1} Q(u) \mathrm{d} u \\
& \lambda_{2}=\frac{1}{2} E\left(X_{2: 2}-X_{1: 2}\right)=\int_{0}^{1} Q(u)(2 u-1) \mathrm{d} u \\
& \lambda_{3}=\frac{1}{3} E\left(X_{3: 3}-2 X_{2: 3}+X_{1: 3}\right)=\int_{0}^{1} Q(u)\left(6 u^{2}-6 u+1\right) \mathrm{d} u \\
& \lambda_{4}=\frac{1}{4} E\left(X_{4: 4}-3 X_{3: 4}+3 X_{2: 4}-X_{1: 4}\right)=\int_{0}^{1} Q(u)\left(20 u^{3}-30 u^{2}+12 u-1\right) \mathrm{d} u
\end{aligned}
$$

while $\lambda_{1}$ and $\lambda_{2}$ can be regarded as measures location and scale, population L-skewness and L-kurtosis are defined as ratios of L-moments:

$$
\begin{aligned}
S K_{5} & =\frac{\lambda_{3}}{\lambda_{2}} \\
K R_{5}^{\prime} & =\frac{\lambda_{4}}{\lambda_{2}}
\end{aligned}
$$

for a standard Normal variate $K R_{5}^{\prime}=0.123$, hence the excess L-kurtosis is $K R_{5}=K R_{5}^{\prime}-$ 0.123. Hosking (1992) showed that L-skewness and L-kurtosis identify deviations from Normality better than conventional moment-based measures. L-moment ratios are bounded: $\left|\lambda_{r} / \lambda_{2}\right|<1$ for $r \geq 3$. The boundedness of $S K_{5}$ and $K R_{5}^{\prime}$ makes their interpretation easier than conventional skewness and kurtosis that can take arbitrarily large values.

Elamir and Seheult (2003) generalized L-moments to distributions that do not posses finite mean: a case in point are Cauchy random variables. Since this approach relies on trimmed samples, they are more robust to outliers than L-moments. Trimmed L-moments (TL-moments), denoted as $\lambda_{r}^{(s, k)}$, are linear functions of expectations of order statistics that exclude the $s$ smallest and/or the $k$ largest order statistics of the sample. In the empirical analysis we set $s=k=1$, therefore we eliminate the largest and smallest observations in

\footnotetext{
${ }^{6}$ Notice that the notation relies on the so-called "conceptual sample", that is different from the sample used for inference (see e.g. Elamir and Seheult, 2003, p. 300). For instance, the population mean is defined in terms of a conceptual sample of size one, therefore $\mu \equiv E(X)=E\left(X_{1: 1}\right)$. Similarly, the population variance is defined in terms of conceptual sample of size two. More generally, the $r$-th moment is defined in terms of a conceptual sample of size $r$.
} 
the sample. The TL-skewness and TL-kurtosis are:

$$
\begin{aligned}
S K_{6} & =\frac{\lambda_{3}^{(1,1)}}{\lambda_{2}^{(1,1)}} \\
K R_{6}^{\prime} & =\frac{\lambda_{4}^{(1,1)}}{\lambda_{2}^{(1,1)}}
\end{aligned}
$$

one drawback of trimming is that, contrarily to $S K_{5}$ and $K R_{5}^{\prime}, S K_{6}$ and $K R_{6}^{\prime}$ are not bounded in the $(-1,1)$ interval. Using results in Hosking (2007) we can show that $\left|S K_{6}\right| \leq$ 1.11 and $\left|K R_{6}^{\prime}\right| \leq 1.25$. For a standard Normal variate $K R_{6}^{\prime}=0.062$, hence the excess TL-kurtosis is $K R_{6}=K R_{6}^{\prime}-0.062$. Thomas (2009) studied tests of symmetry based on $S K_{2}, S K_{3}, S K_{5}$ and $S K_{6}$ and showed that TL-skewness yields the most reliable confidence intervals and tests. Further details, as well as a discussion of the estimation of L- and TL-moments are presented in Section C of the Appendix.

\section{Simulations}

In this section we present simulation results for six alternative distributions both for iid samples and for serially correlated data generating processes. Next, we discuss three additional Monte Carlo designs used to assess the robustness different measures of skewness and kurtosis to outliers. While the design based on iid samples might look simplistic, it used mimic the situation in which regression residuals are analyzed. Nevertheless, we stress that the focus of the paper is on unconditional skewness and kurtosis, therefore we do not directly analyze the effects of filtering the series with a model. See Bai and Ng (2005) and Kilian and Demiroglu (2000) for details on this issue. In Section 3.3 we assess the impact of serial correlation on the set of estimators considered in this paper.

\subsection{Monte Carlo design}

We design our Monte Carlo experiments following Kim and White (2004) and Bonato (2011). We generate $M=1000$ random samples of size $T=50,250,500,1000,2500,5000$ for each design of the simulation analysis. Samples of these dimensions are chosen to mimic the size 
of time series used in macroeconometrics or financial econometrics. For instance, a sample of size $T=50$ is representative of applications that rely on yearly macroeconomic data, while $T=5000$ is typical when dealing with daily or weekly financial returns. In Section 3.2 we focus on three symmetric and three asymmetric distributions. The three symmetric distributions are: the standard Normal and the Student's $t$ distribution with 10 and 5 degrees of freedom. The asymmetric ones are: the Log-Normal distribution with parameters $\mu=1$ and $\sigma=0.4$, the unit Exponential distribution and the $\chi^{2}$ distribution with 3 degrees of freedom. Notice that these distributions have very different shapes and have been used in a variety of financial applications (see e.g. Bollerslev, 1987; Engle and Gallo, 2006); as for their parameters, we select the same as those in Kim and White (2004) and Bonato (2011) to directly compare our results with the literature. A figure showing the probability density functions of these distributions appear in Section D.1 of the Appendix.

\subsubsection{Outliers}

The sample distributions of macroeconomic and financial time series are often asymmetric and heavy-tailed. We thus consider three additional simulation designs aimed at capturing the impact of outliers on the properties of skewness and excess kurtosis estimators.

In a first set of experiments we contaminate draws from a standard Normal distribution with a single outlier. Specifically, we use two designs in which we inject a single, extremely low, value in a standard Normal variate. Following Kim and White (2004), these outliers are calibrated on the empirical distribution of daily log-returns on the S\&P500 index to mimic the stock market crashes of 1987 and 2008. Notice that we do not rely on a closed-form probability density function for the contaminated Normal (see e.g. Gleason, 1993), but we add a single outlier to draws from a standard Normal distribution as detailed in Appendix A.1. This set of simulations aim only at assessing the degree of sensitivity to outliers, but cannot be used to quantify the bias of different estimators. For this reason we do not report these distributions in Table 1. 
Table 1: Values of skewness and excess kurtosis for various distributions

\begin{tabular}{|c|c|c|c|c|c|c|c|c|}
\hline \multicolumn{9}{|c|}{ (a) Skewness measures $\left(S K_{i}\right)$} \\
\hline & \multicolumn{2}{|c|}{$\mathrm{N}(0,1)$} & $t_{10}$ & $t_{5}$ & $\log \mathrm{N}$ & $\operatorname{Exp}(1)$ & $\chi_{(3)}^{2}$ & N-mix \\
\hline$S K_{1}$ & \multicolumn{2}{|c|}{0.000} & 0.000 & 0.000 & 1.322 & 2.000 & 1.633 & -2.271 \\
\hline$S K_{2}$ & \multicolumn{2}{|c|}{0.000} & 0.000 & 0.000 & 0.134 & 0.262 & 0.203 & 0.000 \\
\hline$S K_{3}$ & \multicolumn{2}{|c|}{0.000} & 0.000 & 0.000 & 0.247 & 0.443 & 0.356 & -0.009 \\
\hline$S K_{4}$ & \multicolumn{2}{|c|}{0.000} & 0.000 & 0.000 & 0.185 & 0.307 & 0.259 & -0.007 \\
\hline$S K_{5}$ & \multicolumn{2}{|c|}{0.000} & 0.000 & 0.000 & 0.194 & 0.333 & 0.272 & -0.013 \\
\hline$S K_{6}$ & \multicolumn{2}{|c|}{0.000} & 0.000 & 0.000 & 0.121 & 0.222 & 0.176 & -0.001 \\
\hline \multicolumn{9}{|c|}{ (b) Excess kurtosis measures $\left(K R_{i}\right)$} \\
\hline & \multicolumn{2}{|c|}{$\mathrm{N}(0,1)$} & & & & & & \\
\hline & $K R_{j}^{\prime}$ & $K R_{j}$ & $t_{10}$ & $t_{5}$ & $\log \mathrm{N}$ & $\operatorname{Exp}(1)$ & $\chi_{(3)}^{2}$ & N-mix \\
\hline$K R_{1}$ & 3.000 & 0.000 & 1.000 & 6.000 & 3.260 & 6.000 & 4.000 & 52.575 \\
\hline$K R_{2}$ & 1.233 & 0.000 & 0.044 & 0.094 & 0.044 & 0.073 & 0.042 & 0.001 \\
\hline$K R_{3}$ & 2.585 & 0.000 & 0.200 & 0.460 & 0.187 & 0.279 & 0.168 & 0.088 \\
\hline$K R_{4}$ & 2.906 & 0.000 & 0.278 & 0.632 & 0.268 & 0.429 & 0.248 & 0.009 \\
\hline$K R_{5}$ & 0.123 & 0.000 & 0.032 & 0.071 & 0.030 & 0.044 & 0.027 & 0.014 \\
\hline$K R_{6}$ & 0.062 & 0.000 & 0.013 & 0.029 & 0.013 & 0.021 & 0.012 & 0.000 \\
\hline
\end{tabular}

Notes: distributions are denoted as follows: " $\mathrm{N}(0,1)$ " = standard Normal, " $t_{\nu}$ " = Student's t with $\nu$ degrees of freedom (dof), "Log N" = Log $\operatorname{Normal}(1,0.4)$, "Exp(1)" = Exponential, $\chi_{(\nu)}^{2}=\chi^{2}$ with 3 dof, "N-mix" = Normal mixture. In panel $(b)$ the column headed $K R_{j}^{\prime}$ reports the value of the kurtosis for the standard Normal distribution, while the remaining columns show the kurtosis measure for a given distribution minus the corresponding theoretical value for the standard Normal distribution. For details on the Monte Carlo design see Section A.1 of the Appendix.

Although the previous design is included to allow comparison with Bonato (2011) and Kim and White (2004), it will by definition favor measures of skewness and kurtosis based on TL-moments simply because the minimum and maximum are trimmed. We thus rely also on a Normal mixture distribution with parameters calibrated using the empirical distribution of daily log-returns on the S\&P500 index. This approach also follows that of Kim and White $(2004$, p. 68) and leads to a mixture of the following two distributions: $N(0,1)$ and $N(-7,10)$ with the first occurring with probability $p=.9988$. Notice that the second regime captures stock market crashes; moreover, since in this case nothing prevents to have more than one outlier of the same sign, results are not biased toward favoring estimators of skewness and kurtosis based on TL-moments. As shown in Table 1 the theoretical values of the conventional skewness and excess kurtosis of this distribution are -2.27 and 52.58 . We stress that, contrarily to the single outlier case, this design can be used to quantify the bias of different estimators. 


\subsubsection{Evaluation of estimators}

We evaluate estimators of skewness and excess kurtosis in terms of bias and root mean squared error. The bias is defined as $B_{j}=E\left(\theta_{j}-\theta_{j}^{(0)}\right)$ where $\theta_{j}^{(0)}$ represents the true value of the $j$-th measure of skewness or excess kurtosis as reported in Table 1 . The Monte Carlo estimates of the bias are: $\hat{B}_{j}=\overline{\hat{\theta}}_{j}-\theta_{j}^{(0)}$ where $\overline{\hat{\theta}}_{j}=M^{-1} \sum_{m=1}^{M} \hat{\theta}_{j, m}$ and $\hat{\theta}_{j, m}$ is the sample estimate obtained in the $m$-th simulation and $M=1000$ is the number of simulations.

We stress that the magnitude of the bias of different estimators of skewness and kurtosis cannot be directly compared, because some of them are constrained to lie in a finite interval (e.g. L-skewness and kurtosis), while others can take on any real value (e.g. standard measures of skewness and kurtosis). Nevertheless, providing an estimate of the bias and assessing whether and how quickly vanishes as the sample size grows provides useful information to evaluate different estimators.

A similar warning applies also when we assess the root mean squared error (RMSE) of different estimators. This is defined as: $R M S E_{j}=\sqrt{E\left(\theta_{j}-\theta_{j}^{(0)}\right)^{2}}$. To ease the comparison, we rely on RMSE ratios defined as: $R M S E_{j} / R M S E_{1}$, where $R M S E_{1}$ identifies the RMSE of the conventional moment-based skewness or excess kurtosis. Because different estimators have different scales, several caveats should be kept in mind when interpreting RMSE ratios. First, a RMSE ratio lower than one signals that the $j$-th estimator is more tightly clustered around its population value than the conventional measures $\left(S K_{1}\right.$ or $\left.K R_{1}\right)$ only if they are both unbounded (i.e. this interpretation applies to $S K_{4}$ and $K R_{2}-K R_{4}$ ). Second, in the case of skewness measures, we can can only compare the relative change in RMSE ratios of $S K_{2}, S K_{3}$ and $S K_{5}$ with each other (i.e. all of them lie in the (-1,1) interval), but we cannot compare them neither with the RMSE ratio of $S K_{4}$ (i.e. it is not bounded to lie in a closed interval) nor with that of $S K_{6}$ (i.e. it is bounded on an closed interval of different length). As for excess kurtosis, we can only compare the RMSE ratios of $K R_{2}$, $K R_{3}$ and $K R_{4}$; in fact, $K R_{5}$ and $K R_{6}$ are bounded to lie in closed intervals with length. 


\subsection{Results for iid samples}

In this section we evaluate the performance of measures of skewness and excess kurtosis for iid random samples generated from the the standard Normal, Student's $t$, Log-Normal, Exponential and $\chi^{2}$.

Table 2 shows that the bias of $S K_{1}$ increases as we move away from the Normal distribution, especially for small samples and for asymmetric distributions. This fact is clearly visible in Figure 1 where, for each data generating process and sample size, we summarize the sampling distribution $S K_{1}$ with a boxplot. ${ }^{7}$

For the Log-Normal, Exponential and $\chi^{2}$ distributions there is a substantial downward bias, that is still visible even in samples of 250 observations. Numbers in Figure 1 represent observations that are greater than $Q_{0.75}+I Q R$ or lower than $Q_{0.25}+I Q R$, where $I Q R$ is the interquartile range. These numbers show that the dispersion of $S K_{1}$ increases when we move from the Normal to the Student's $t$ distribution and also when considering asymmetric distributions.

As for the robust measures, Table 2 conveys two main messages. First the bias of $S K_{2^{-}}$ $S K_{6}$ is in most cases negligible; second, the L-moment skewness, $S K_{5}$, is always associated with the lowest RMSE ratio when compared with that of $S K_{2}$ and $S K_{3}$.

\footnotetext{
${ }^{7}$ Boxplots with the sampling distributions of all estimators are shown in Appendix D.
} 
Table 2: Bias and RMSE ratio of skewness estimators

\begin{tabular}{|c|c|c|c|c|c|c|}
\hline$T$ & 50 & 250 & 500 & 1000 & 2500 & 5000 \\
\hline \multicolumn{7}{|c|}{ (a) $\mathrm{N}(0,1)$} \\
\hline$S K_{1}$ & $0.003(1.000)$ & $0.007(1.000)$ & $0.002(1.000)$ & $0.001(1.000)$ & $0.001(1.000)$ & $0.000(1.000)$ \\
\hline$S K_{2}$ & $-0.009(0.575)$ & $0.000(0.505)$ & $0.001(0.583)$ & $-0.001(0.518)$ & $-0.001(0.564)$ & $0.001(0.569)$ \\
\hline$S K_{3}$ & $-0.002(0.403)$ & $-0.000(0.366)$ & $0.002(0.402)$ & $-0.000(0.364)$ & $-0.000(0.394)$ & $0.001(0.392)$ \\
\hline$S K_{4}$ & $-0.001(0.321)$ & $-0.000(0.292)$ & $0.001(0.321)$ & $-0.000(0.290)$ & $-0.000(0.314)$ & $0.001(0.313)$ \\
\hline$S K_{5}$ & $0.001(0.197)$ & $0.001(0.179)$ & $0.001(0.181)$ & $0.000(0.174)$ & $0.000(0.176)$ & $0.000(0.174)$ \\
\hline$S K_{6}$ & $-0.001(0.179)$ & $-0.000(0.157)$ & $0.000(0.165)$ & $-0.000(0.150)$ & $0.000(0.157)$ & $0.000(0.157)$ \\
\hline \multicolumn{7}{|c|}{ (b) $t_{10}$} \\
\hline$S K_{1}$ & $-0.007(1.000)$ & $-0.007(1.000)$ & $-0.001(1.000)$ & $-0.008(1.000)$ & $0.001(1.000)$ & $-0.000(1.000)$ \\
\hline$S K_{2}$ & $0.000(0.394)$ & $0.003(0.315)$ & $-0.003(0.283)$ & $-0.001(0.270)$ & $-0.001(0.285)$ & $-0.001(0.282)$ \\
\hline$S K_{3}$ & $-0.001(0.271)$ & $0.001(0.219)$ & $-0.003(0.198)$ & $-0.002(0.196)$ & $-0.000(0.201)$ & $-0.001(0.196)$ \\
\hline$S K_{4}$ & $-0.001(0.211)$ & $0.001(0.169)$ & $-0.002(0.154)$ & $-0.001(0.151)$ & $-0.000(0.156)$ & $-0.000(0.152)$ \\
\hline$S K_{5}$ & $-0.000(0.153)$ & $-0.001(0.116)$ & $-0.000(0.106)$ & $-0.001(0.108)$ & $-0.000(0.108)$ & $-0.000(0.103)$ \\
\hline$S K_{6}$ & $0.000(0.116)$ & $0.000(0.095)$ & $-0.001(0.083)$ & $-0.001(0.086)$ & $-0.000(0.088)$ & $-0.000(0.083)$ \\
\hline \multicolumn{7}{|c|}{ (c) $t_{5}$} \\
\hline$S K_{1}$ & $0.012(1.000)$ & 0.004 & $0.000(1.000)$ & $-0.012(1.000)$ & $-0.013(1.000)$ & $-0.023(1.000)$ \\
\hline$S K_{2}$ & $0.009(0.235)$ & $-0.005(0.097)$ & $-0.003(0.090)$ & $0.002(0.086)$ & $0.001(0.075)$ & $0.000(0.039)$ \\
\hline$S K_{3}$ & $0.005(0.171)$ & $-0.004(0.072)$ & $-0.002(0.065)$ & $-0.000(0.064)$ & $-0.000(0.054)$ & $0.000(0.029)$ \\
\hline$S K_{4}$ & $0.003(0.128)$ & $-0.003(0.053)$ & $-0.002(0.047)$ & $-0.000(0.047)$ & $-0.000(0.039)$ & $0.000(0.021)$ \\
\hline$S K_{5}$ & $0.001(0.115)$ & $-0.001(0.051)$ & $-0.001(0.044)$ & $-0.001(0.043)$ & $-0.001(0.037)$ & $0.000(0.019)$ \\
\hline$S K_{6}$ & $0.001(0.075)$ & $-0.001(0.031)$ & $-0.001(0.029)$ & $0.000(0.028)$ & $-0.000(0.024)$ & $0.000(0.013)$ \\
\hline \multicolumn{7}{|c|}{ (d) $\log -\mathrm{N}(1,0.4)$} \\
\hline$S K_{1}$ & $-0.235(1.000)$ & $-0.072(1.000)$ & $-0.046(1.000)$ & $-0.025(1.000)$ & $-0.009(1.000)$ & $-0.006(1.000)$ \\
\hline$S K_{2}$ & $-0.012(0.310)$ & $-0.003(0.238)$ & $-0.004(0.221)$ & $-0.002(0.196)$ & $0.000(0.185)$ & $-0.001(0.179)$ \\
\hline$S K_{3}$ & $-0.007(0.214)$ & $-0.004(0.160)$ & $-0.003(0.150)$ & $-0.002(0.137)$ & $-0.000(0.124)$ & $-0.000(0.122)$ \\
\hline$S K_{4}$ & $-0.006(0.156)$ & $-0.003(0.116)$ & $-0.002(0.108)$ & $-0.001(0.098)$ & $-0.000(0.089)$ & $-0.000(0.088)$ \\
\hline$S K_{5}$ & $-0.001(0.118)$ & $-0.001(0.084)$ & $-0.001(0.079)$ & $-0.001(0.073)$ & $-0.000(0.064)$ & $0.000(0.066)$ \\
\hline$S K_{6}$ & $-0.019(0.102)$ & $-0.003(0.072)$ & $-0.001(0.067)$ & $-0.001(0.060)$ & $-0.000(0.054)$ & $-0.000(0.055)$ \\
\hline \multicolumn{7}{|c|}{ (e) $\operatorname{Exp}(1)$} \\
\hline$S K_{1}$ & $-0.369(1.000)$ & -0.0 & $3(1.000)$ & $-0.026(1.000)$ & $-0.002(1.000)$ & $-0.007(1.000)$ \\
\hline$S K_{2}$ & $-0.011(0.256)$ & $-0.004(0.191)$ & $-0.003(0.188)$ & $0.001(0.165)$ & $-0.000(0.155)$ & $-0.000(0.161)$ \\
\hline$S K_{3}$ & $-0.013(0.176)$ & $-0.004(0.126)$ & $-0.003(0.127)$ & $0.001(0.111)$ & $-0.001(0.106)$ & $-0.000(0.111)$ \\
\hline$S K_{4}$ & $-0.005(0.117)$ & $-0.002(0.082)$ & $-0.001(0.081)$ & $0.001(0.072)$ & $-0.000(0.067)$ & $-0.000(0.072)$ \\
\hline$S K_{5}$ & $-0.005(0.098)$ & $-0.000(0.068)$ & $-0.001(0.067)$ & $0.000(0.058)$ & $-0.000(0.057)$ & $-0.000(0.061)$ \\
\hline$S K_{6}$ & $-0.031(0.094)$ & $-0.003(0.060)$ & $-0.002(0.060)$ & $-0.000(0.051)$ & $-0.000(0.049)$ & $-0.000(0.052)$ \\
\hline \multicolumn{7}{|c|}{$(f) \chi_{(3)}^{2}$} \\
\hline$S K_{1}$ & $-0.291(1.000)$ & $-0.060(1.000)$ & $-0.040(1.000)$ & $-0.023(1.000)$ & $-0.001(1.000)$ & $-0.007(1.000)$ \\
\hline$S K_{2}$ & $-0.019(0.299)$ & $-0.006(0.260)$ & $-0.003(0.222)$ & $0.001(0.228)$ & $0.001(0.209)$ & $-0.001(0.200)$ \\
\hline$S K_{3}$ & $-0.018(0.207)$ & $-0.004(0.171)$ & $-0.003(0.150)$ & $0.000(0.155)$ & $0.001(0.141)$ & $-0.001(0.137)$ \\
\hline$S K_{4}$ & $-0.012(0.145)$ & $-0.003(0.118)$ & $-0.002(0.102)$ & $0.000(0.106)$ & $0.000(0.095)$ & $-0.000(0.094)$ \\
\hline$S K_{5}$ & $-0.007(0.111)$ & $-0.001(0.087)$ & $-0.002(0.077)$ & $-0.000(0.079)$ & $0.000(0.071)$ & $-0.000(0.069)$ \\
\hline$S K_{6}$ & $-0.026(0.104)$ & $-0.004(0.081)$ & $-0.002(0.067)$ & $-0.000(0.068)$ & $0.000(0.062)$ & $-0.000(0.061)$ \\
\hline
\end{tabular}

Notes: for each sample size $T$ and distribution the table shows the bias $\left(B_{j}\right)$ and the Root Mean Squared Error (RMSE) ratio of the estimator (in brackets). RMSE ratios are defined as: $R M S E_{j} / R M S E_{1}$, where $R M S E_{1}$ identifies the RMSE of the method-of-moments estimator. Estimates of $B_{j}$ and $R M S E_{j}$ are based on $M=1000$ Monte Carlo simulations. See Section 3.1.2 for details on the interpretation of results in the table. 
Table 3: Bias and RMSE ratio of excess kurtosis estimators

\begin{tabular}{|c|c|c|c|c|c|c|}
\hline$T$ & 50 & 250 & 500 & 1000 & 2500 & 5000 \\
\hline \multicolumn{7}{|c|}{ (a) $\mathrm{N}(0,1)$} \\
\hline$K R_{1}$ & $-0.119(1.000)$ & $-0.032(1.000)$ & $-0.019(1.000)$ & $-0.002(1.000)$ & $-0.000(1.000)$ & $-0.002(1.000)$ \\
\hline$K R_{2}$ & $0.019(0.425)$ & $0.006(0.382)$ & $0.001(0.388)$ & $0.002(0.353)$ & $0.000(0.377)$ & $0.000(0.368)$ \\
\hline$K R_{3}$ & $-0.074(0.407)$ & $-0.017(0.368)$ & $-0.009(0.365)$ & $-0.003(0.343)$ & $-0.007(0.363)$ & $-0.009(0.378)$ \\
\hline$K R_{4}$ & $0.035(0.990)$ & $-0.014(0.825)$ & $0.002(0.830)$ & $0.013(0.786)$ & $0.001(0.813)$ & $0.003(0.795)$ \\
\hline$K R_{5}$ & $0.000(0.075)$ & $-0.001(0.064)$ & $-0.000(0.063)$ & $0.000(0.059)$ & $0.000(0.061)$ & $0.000(0.061)$ \\
\hline$K R_{6}$ & $-0.148(0.251)$ & $-0.010(0.062)$ & $-0.003(0.056)$ & $-0.001(0.048)$ & $-0.000(0.052)$ & $0.000(0.050)$ \\
\hline \multicolumn{7}{|c|}{ (b) $t_{10}$} \\
\hline$K R_{1}$ & $-0.534(1.000)$ & $-0.101(1.000)$ & $-0.057(1.000)$ & $-0.027(1.000)$ & $-0.025(1.000)$ & $0.007(1.000)$ \\
\hline$K R_{2}$ & $0.036(0.227)$ & $0.005(0.105)$ & $0.003(0.090)$ & $0.002(0.100)$ & $0.000(0.095)$ & $0.001(0.082)$ \\
\hline$K R_{3}$ & $-0.102(0.249)$ & $-0.017(0.119)$ & $-0.010(0.102)$ & $-0.010(0.114)$ & $-0.016(0.120)$ & $-0.022(0.110)$ \\
\hline$K R_{4}$ & $0.110(0.617)$ & $-0.013(0.258)$ & $0.006(0.220)$ & $0.005(0.245)$ & $0.003(0.241)$ & $0.002(0.199)$ \\
\hline$K R_{5}$ & $-0.001(0.042)$ & $-0.000(0.019)$ & $-0.000(0.016)$ & $0.000(0.018)$ & $0.000(0.018)$ & $0.000(0.015)$ \\
\hline$K R_{6}$ & $-0.155(0.126)$ & $-0.012(0.018)$ & $-0.003(0.013)$ & $-0.001(0.014)$ & $-0.000(0.013)$ & $0.000(0.011)$ \\
\hline \multicolumn{7}{|c|}{ (c) $t_{5}$} \\
\hline$K R_{1}$ & $-4.436(1.000)$ & $-2.249(1.000)$ & $-1.944(1.000)$ & $-1.892(1.000)$ & $-1.536(1.000)$ & $-0.614(1.000)$ \\
\hline$K R_{2}$ & $0.034(0.058)$ & $0.009(0.015)$ & $-0.000(0.013)$ & $0.003(0.011)$ & $0.000(0.008)$ & $-0.001(0.002)$ \\
\hline$K R_{3}$ & $-0.133(0.077)$ & $-0.019(0.023)$ & $-0.011(0.020)$ & $-0.018(0.016)$ & $-0.040(0.015)$ & $-0.056(0.005)$ \\
\hline$K R_{4}$ & $0.247(0.213)$ & $-0.003(0.044)$ & $0.009(0.039)$ & $0.005(0.032)$ & $-0.000(0.024)$ & $0.001(0.006)$ \\
\hline$K R_{5}$ & $-0.003(0.012)$ & $0.000(0.003)$ & $0.000(0.003)$ & $0.000(0.002)$ & $-0.000(0.002)$ & $-0.000(0.000)$ \\
\hline$K R_{6}$ & $-0.164(0.033)$ & $-0.013(0.003)$ & $-0.004(0.002)$ & $-0.001(0.002)$ & $-0.000(0.001)$ & $-0.000(0.000)$ \\
\hline \multicolumn{7}{|c|}{$(d) \log -\mathrm{N}(1,0.4)$} \\
\hline$K R_{1}$ & $-1.614(1.000)$ & $-0.583(1.000)$ & $-0.388(1.000)$ & $-0.202(1$. & $-0.057(1.000)$ & $-0.062(1.000)$ \\
\hline$K R_{2}$ & $0.031(0.093)$ & $0.013(0.053)$ & $0.004(0.042)$ & $0.002(0.033)$ & $-0.001(0.027)$ & $0.000(0.029)$ \\
\hline$K R_{3}$ & $-0.088(0.110)$ & $-0.013(0.064)$ & $-0.011(0.054)$ & $-0.012(0.041)$ & $-0.016(0.038)$ & $-0.020(0.040)$ \\
\hline$K R_{4}$ & $-0.241(0.212)$ & $-0.067(0.131)$ & $-0.027(0.108)$ & $-0.007(0.087)$ & $-0.008(0.073)$ & $-0.001(0.073)$ \\
\hline$K R_{5}$ & $0.001(0.019)$ & $0.000(0.010)$ & $-0.000(0.008)$ & $-0.000(0.007)$ & $-0.000(0.006)$ & $-0.000(0.006)$ \\
\hline$K R_{6}$ & $-0.155(0.051)$ & $-0.011(0.008)$ & $-0.003(0.006)$ & $-0.001(0.005)$ & $-0.000(0.004)$ & $-0.000(0.004)$ \\
\hline \multicolumn{7}{|c|}{ (e) $\operatorname{Exp}(1)$} \\
\hline$K R_{1}$ & $-2.889(1.000)$ & $-0.756(1.000)$ & $-0.603(1.000)$ & $-0.264(1.000)$ & $-0.012(1.000)$ & $-0.067(1.000)$ \\
\hline$K R_{2}$ & $0.024(0.069)$ & $0.008(0.037)$ & $0.005(0.032)$ & $0.000(0.027)$ & $-0.000(0.025)$ & $0.001(0.026)$ \\
\hline$K R_{3}$ & $-0.114(0.094)$ & $-0.012(0.050)$ & $-0.013(0.043)$ & $-0.010(0.036)$ & $-0.015(0.035)$ & $-0.021(0.040)$ \\
\hline$K R_{4}$ & $-0.418(0.184)$ & $-0.098(0.105)$ & $-0.041(0.094)$ & $-0.008(0.075)$ & $-0.006(0.075)$ & $0.003(0.078)$ \\
\hline$K R_{5}$ & $-0.003(0.015)$ & $0.000(0.008)$ & $-0.000(0.007)$ & $0.000(0.005)$ & $0.000(0.005)$ & $0.000(0.005)$ \\
\hline$K R_{6}$ & $-0.160(0.036)$ & $-0.012(0.006)$ & $-0.003(0.005)$ & $-0.001(0.004)$ & $-0.000(0.004)$ & $0.000(0.004)$ \\
\hline \multicolumn{7}{|c|}{$(f) \chi_{(3)}^{2}$} \\
\hline$K R_{1}$ & $-1.917(1.000)$ & $-0.531(1.000)$ & $-0.299(1.000)$ & $-0.213(1.000)$ & $-0.003(1.000)$ & $-0.048(1.000)$ \\
\hline$K R_{2}$ & $0.044(0.091)$ & $0.001(0.057)$ & $-0.002(0.047)$ & $0.000(0.045)$ & $0.002(0.036)$ & $0.001(0.030)$ \\
\hline$K R_{3}$ & $-0.108(0.109)$ & $-0.008(0.070)$ & $-0.015(0.058)$ & $-0.010(0.057)$ & $-0.012(0.046)$ & $-0.018(0.043)$ \\
\hline$K R_{4}$ & $-0.328(0.207)$ & $-0.093(0.147)$ & $-0.058(0.119)$ & $-0.008(0.119)$ & $-0.008(0.096)$ & $-0.002(0.081)$ \\
\hline$K R_{5}$ & $-0.002(0.018)$ & $0.001(0.011)$ & $-0.001(0.009)$ & $-0.000(0.009)$ & $0.000(0.007)$ & $-0.000(0.006)$ \\
\hline$K R_{6}$ & $-0.153(0.046)$ & $-0.011(0.009)$ & $-0.004(0.007)$ & $-0.001(0.006)$ & $-0.000(0.005)$ & $-0.000(0.004)$ \\
\hline
\end{tabular}

Notes: see notes to Table 2. 
Figure 1: Sample distribution of $S K_{1}$
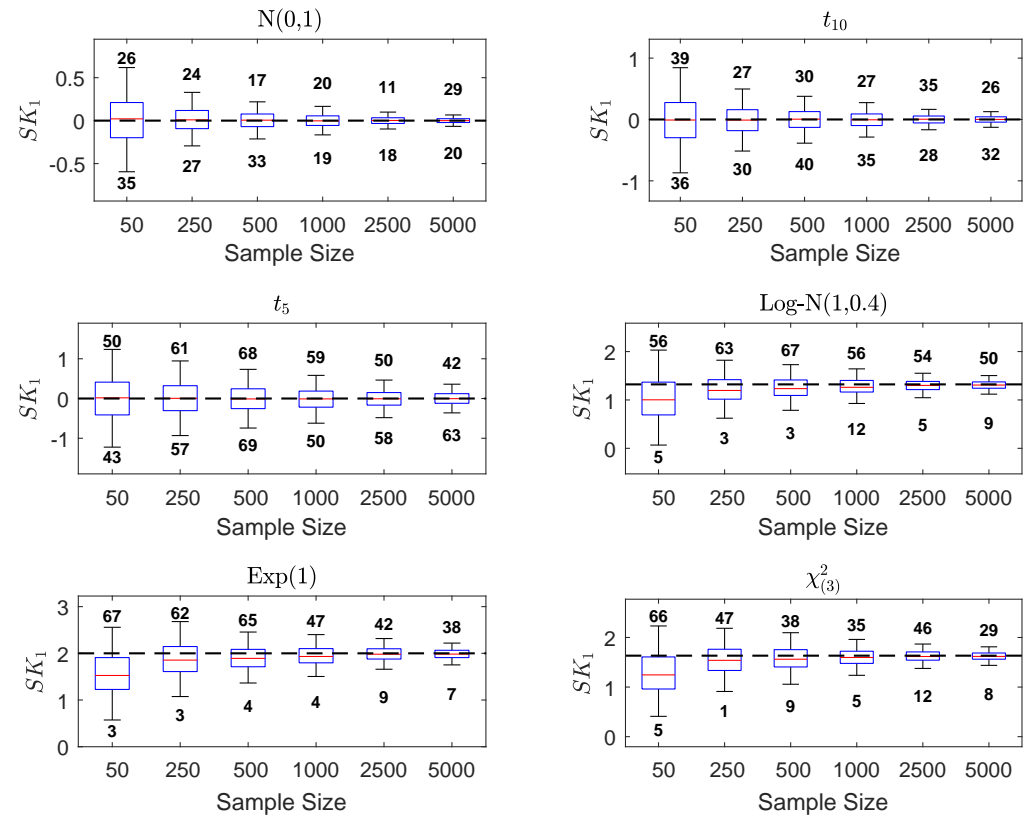

Notes: for each distribution and sample size (reported on the $x$-axis) the figure represents the sampling distribution of the estimator with a boxplot. The line in the middle of the box is the median, the size of the box is proportional to the interquartile range $(I Q R)$, namely the distance between the 75 -th $\left(Q_{0.75}\right)$ and 25 -th percentile $Q_{0.25}$. The bottom (top) external line, known as whisker, is drawn in correspondence of $Q_{0.25}-I Q R\left(Q_{0.75}+I Q R\right)$. Numbers outside the two whiskers count the observations lower (greater) than the bottom (top) whisker. 
Figure 2: Sample distribution of $K R_{1}$
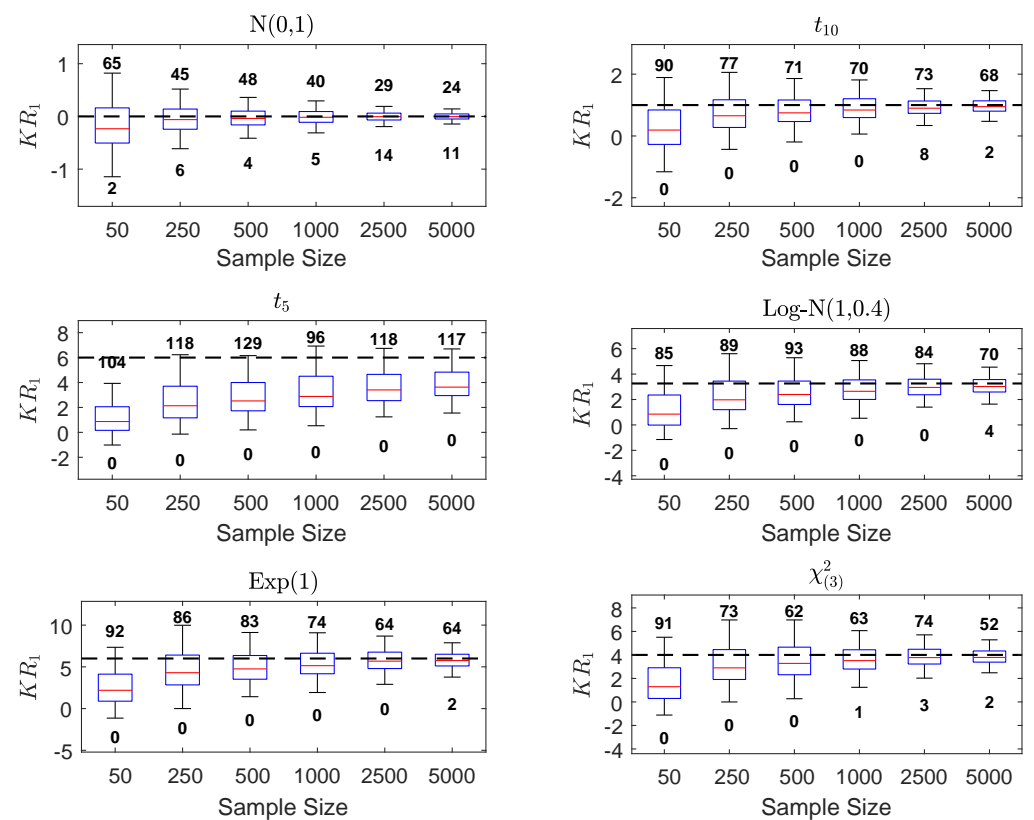

Notes: see notes to Figure 1.

Moreover, notice that trimming leads to a bias in the case of samples of size 50 drawn from asymmetric distributions. As it can been seen, this does not happen for L-skewness, $S K_{5}$. To sum up, measures based on L- or TL-moments represent the best choice in terms of bias; in addition, $S K_{5}$ seems to be competitive also in terms of RMSE when compared to some of the alternative robust estimators.

Table 3 and Figure 2 highlight that the conventional measure of excess kurtosis $K R_{1}$ is severely biased, especially in small samples. For the Student's $t_{5}$ distribution the downward bias can be visually assessed in Figure 2, even in a sample of 5000 observations. Moreover, as in the case of the method-of-moments skewness measure, $K R_{1}$ is always associated with the highest RMSE when compared with $K R_{2}-K R_{4}$. In samples of size up to 250 the best options in terms of bias are $K R_{2}$ and $K R_{5}$ : in both cases the bias vanishes as we move from 50 to 250 observations. On the contrary, when the underlying distribution is asymmetric, the remaining measures are downward biased even in samples of 250 observations. Much like in the case of skewness, trimming leads to a deterioration of the performance of the estimator based on TL-moments; in fact, irrespective of the distribution, $K R_{6}$ displays a severe bias in samples of size 50 . 


\subsection{Serial correlation}

Up to now we have analysed iid samples, but macroeconomic and financial time series are typically serially correlated and often highly persistent. For this reason, we now briefly assess the effects of serial correlation on the measures of skewness and excess kurtosis under investigation. We do so by focusing on two Autoregressive models of order one, AR(1), with different error distributions and degrees of persistence. ${ }^{8}$ For the sake of brevity, we present only three distributions: the standard Normal, Student's $t_{5}$ and the Log-Normal distribution. In Tables 4 and 5 we consider an AR(1) model with autoregressive parameter equal to 0.9 . Results for an AR(1) model with autoregressive parameter 0.5 appear in Appendix D.

Moving from the iid simulation design to the AR(1) data generating process, we see that the bias of all skewness and excess kurtosis measures increases in absolute terms. As for skewness, L-moments often outperform $S K_{2}$ and $S K_{3}$ for what concerns the RMSE ratio. As the sample size increases, for non-Normal distributions, the bias of $K R_{6}$ tends to vanish more quickly than that of its competitors.

All in all, also in the presence of serial correlation the bias of skewness and kurtosis measures based L- or TL-moments vanishes very quickly as the sample size grows. Table D1 and D2 of the Appendix present results for AR(1) models with autoregressive parameter equal to 0.5. These tables confirm the findings of this section, therefore the main results are not affected by the degree of persistence of the $\mathrm{AR}(1)$ model. The degree of persistence is positively associated with the size of the bias, but does not alter the ranking of estimators.

\footnotetext{
${ }^{8}$ We simulate two zero mean $\operatorname{AR}(1)$ processes with autoregressive parameter $\rho=0.5$ and $\rho=0.9$. If we measure persistence in terms of "speed of mean reversion", we can rely on the half-life to quantify the number of periods needed for the effects of a unit shock to halve. For an $\mathrm{AR}(1)$ model, the half-life is: $H L=\log (0.5) / \log (|\rho|)$. Then, we get $H L=1$ when $\rho=0.5$ and $H L=6.6$ when $\rho=0.9$.
} 
Table 4: Bias and RMSE ratio of skewness estimators - $\operatorname{AR}(1)$ model $(\rho=0.9)$

\begin{tabular}{lcccccc}
\hline$T$ & 50 & 250 & 500 & 1000 & 2500 & 5000 \\
\hline$S K_{1}$ & $0.017(1.000)$ & $-0.008(1.000)$ & $-0.009(1.000)$ & $-0.002(1.000)$ & $0.002(1.000)$ & $0.002(1.000)$ \\
$S K_{2}$ & $0.005(0.522)$ & $0.001(0.363)$ & $0.002(0.336)$ & $-0.000(0.310)$ & $0.001(0.294)$ & $0.001(0.311)$ \\
$S K_{3}$ & $0.006(0.451)$ & $0.000(0.335)$ & $0.000(0.303)$ & $-0.001(0.283)$ & $0.001(0.278)$ & $0.001(0.280)$ \\
$S K_{4}$ & $0.005(0.362)$ & $0.000(0.267)$ & $0.000(0.242)$ & $-0.001(0.226)$ & $0.001(0.222)$ & $0.001(0.223)$ \\
$S K_{5}$ & $0.005(0.236)$ & $-0.001(0.194)$ & $-0.001(0.183)$ & $-0.001(0.177)$ & $0.000(0.173)$ & $0.001(0.170)$ \\
$S K_{6}$ & $0.005(0.225)$ & $-0.001(0.159)$ & $0.001(0.145)$ & $-0.001(0.137)$ & $0.000(0.135)$ & $0.000(0.132)$ \\
\hline \multicolumn{7}{c}{$(c) t_{5}$} \\
\hline$S K_{1}$ & $0.002(1.000)$ & $-0.006(1.000)$ & $0.000(1.000)$ & $0.006(1.000)$ & $-0.003(1.000)$ & $0.003(1.000)$ \\
$S K_{2}$ & $-0.011(0.468)$ & $0.002(0.310)$ & $-0.001(0.270)$ & $-0.002(0.220)$ & $0.002(0.188)$ & $0.001(0.182)$ \\
$S K_{3}$ & $-0.003(0.406)$ & $0.001(0.280)$ & $-0.001(0.248)$ & $-0.000(0.203)$ & $0.001(0.176)$ & $0.001(0.173)$ \\
$S K_{4}$ & $-0.003(0.325)$ & $0.001(0.220)$ & $-0.000(0.195)$ & $-0.000(0.159)$ & $0.001(0.138)$ & $0.001(0.136)$ \\
$S K_{5}$ & $-0.000(0.220)$ & $-0.001(0.171)$ & $-0.000(0.157)$ & $0.000(0.132)$ & $-0.000(0.116)$ & $0.000(0.117)$ \\
$S K_{6}$ & $-0.002(0.194)$ & $-0.000(0.134)$ & $-0.000(0.122)$ & $-0.000(0.099)$ & $0.000(0.086)$ & $0.000(0.084)$ \\
\hline & & & $(d) \log -\mathrm{N}(1,0.4)$ & & \\
\hline$S K_{1}$ & $-1.112(1.000)$ & $-0.976(1.000)$ & $-0.940(1.000)$ & $-0.940(1.000)$ & $-0.925(1.000)$ & $-0.921(1.000)$ \\
$S K_{2}$ & $-0.104(0.209)$ & $-0.095(0.146)$ & $-0.086(0.121)$ & $-0.090(0.110)$ & $-0.090(0.104)$ & $-0.091(0.102)$ \\
$S K_{3}$ & $-0.189(0.226)$ & $-0.170(0.194)$ & $-0.162(0.183)$ & $-0.166(0.181)$ & $-0.166(0.181)$ & $-0.166(0.181)$ \\
$S K_{4}$ & $-0.139(0.174)$ & $-0.124(0.145)$ & $-0.117(0.134)$ & $-0.120(0.132)$ & $-0.120(0.132)$ & $-0.121(0.132)$ \\
$S K_{5}$ & $-0.147(0.150)$ & $-0.132(0.141)$ & $-0.128(0.139)$ & $-0.131(0.140)$ & $-0.130(0.141)$ & $-0.130(0.141)$ \\
$S K_{6}$ & $-0.089(0.110)$ & $-0.082(0.094)$ & $-0.079(0.089)$ & $-0.082(0.089)$ & $-0.081(0.089)$ & $-0.081(0.089)$ \\
\hline
\end{tabular}

Notes: see notes to Table 2. 
Table 5: Bias and RMSE ratio of excess kurtosis estimators - AR(1) model $(\rho=0.9)$

\begin{tabular}{lcccccc}
\hline$T$ & 50 & 250 & 500 & 1000 & 2500 & 5000 \\
\hline$K R_{1}$ & $-0.461(1.000)$ & $-0.167(1.000)$ & $-0.117(1.000)$ & $-0.054(1.000)$ & $-0.018(1.000)$ & $-0.007(1.000)$ \\
$K R_{2}$ & $0.003(0.456)$ & $-0.006(0.311)$ & $0.004(0.274)$ & $0.001(0.261)$ & $0.000(0.214)$ & $0.002(0.245)$ \\
$K R_{3}$ & $-0.258(0.513)$ & $-0.072(0.412)$ & $-0.046(0.389)$ & $-0.020(0.376)$ & $-0.012(0.336)$ & $-0.010(0.351)$ \\
$K R_{4}$ & $-0.252(0.945)$ & $-0.049(0.804)$ & $-0.015(0.756)$ & $0.002(0.726)$ & $-0.001(0.614)$ & $-0.001(0.660)$ \\
$K R_{5}$ & $-0.033(0.093)$ & $-0.010(0.071)$ & $-0.006(0.066)$ & $-0.003(0.063)$ & $-0.001(0.055)$ & $-0.000(0.058)$ \\
$K R_{6}$ & $-0.153(0.212)$ & $-0.012(0.055)$ & $-0.004(0.046)$ & $-0.001(0.044)$ & $-0.000(0.037)$ & $0.000(0.041)$ \\
\hline \multicolumn{7}{c}{$(c) t_{5}$} \\
\hline$K R_{1}$ & $-6.335(1.000)$ & $-5.923(1.000)$ & $-5.744(1.000)$ & $-5.620(1.000)$ & $-5.528(1.000)$ & $-5.496(1.000)$ \\
$K R_{2}$ & $-0.072(0.060)$ & $-0.074(0.032)$ & $-0.073(0.024)$ & $-0.071(0.020)$ & $-0.074(0.016)$ & $-0.076(0.015)$ \\
$K R_{3}$ & $-0.688(0.119)$ & $-0.466(0.087)$ & $-0.417(0.078)$ & $-0.395(0.073)$ & $-0.389(0.071)$ & $-0.383(0.070)$ \\
$K R_{4}$ & $-0.810(0.176)$ & $-0.562(0.126)$ & $-0.523(0.110)$ & $-0.515(0.101)$ & $-0.520(0.097)$ & $-0.513(0.095)$ \\
$K R_{5}$ & $-0.099(0.019)$ & $-0.070(0.013)$ & $-0.063(0.012)$ & $-0.060(0.011)$ & $-0.058(0.011)$ & $-0.057(0.010)$ \\
$K R_{6}$ & $-0.180(0.029)$ & $-0.036(0.008)$ & $-0.027(0.006)$ & $-0.024(0.005)$ & $-0.024(0.005)$ & $-0.024(0.004)$ \\
\hline \multicolumn{7}{c}{$(d) \log -\mathrm{N}(1,0.4)$} \\
\hline$K R_{1}$ & $-3.670(1.000)$ & $-3.234(1.000)$ & $-3.085(1.000)$ & $-3.034(1.000)$ & $-2.958(1.000)$ & $-2.939(1.000)$ \\
$K R_{2}$ & $-0.042(0.086)$ & $-0.039(0.048)$ & $-0.030(0.037)$ & $-0.034(0.028)$ & $-0.037(0.020)$ & $-0.038(0.018)$ \\
$K R_{3}$ & $-0.432(0.138)$ & $-0.238(0.096)$ & $-0.195(0.079)$ & $-0.180(0.070)$ & $-0.168(0.062)$ & $-0.169(0.060)$ \\
$K R_{4}$ & $-0.555(0.222)$ & $-0.302(0.158)$ & $-0.240(0.123)$ & $-0.233(0.105)$ & $-0.228(0.090)$ & $-0.230(0.086)$ \\
$K R_{5}$ & $-0.060(0.023)$ & $-0.036(0.015)$ & $-0.030(0.013)$ & $-0.028(0.011)$ & $-0.026(0.010)$ & $-0.025(0.009)$ \\
$K R_{6}$ & $-0.164(0.045)$ & $-0.023(0.010)$ & $-0.014(0.007)$ & $-0.012(0.006)$ & $-0.011(0.005)$ & $-0.011(0.004)$ \\
\hline
\end{tabular}

Notes: see notes to Table 2 . 


\subsection{Robustness to outliers}

Joint inspection of Figures 3 and 5 highlights that the effects of outlying observations on the sampling distributions of conventional skewness and kurtosis measures depend heavily on the data generating process used in the simulations.

\subsubsection{Normal mixture distribution}

Figure 3 shows that the dispersion of $S K_{1}$ increases with the size of the sample. This happens because the mixture distribution assigns a very low probability to the "market crash regime", that therefore materializes more often in larger samples. Independently of the sample size, Table 6 shows that $S K_{1}$ is upward biased with the size of the bias monotonically decreasing as $T$ increases. On the contrary, the robust measures of skewness are largely unaffected by the presence of very large negative observations generated in the second regime of the mixture. See Figure 4.

Figure 5 shows that $K R_{1}$ is severely downward biased even in samples of size 5000 . The Normal mixture data generating process proves challenging also for $K R_{3}$ that always displays a negative bias. On the contrary, $K R_{2}, K R_{4}$ and $K R_{5}$ are centered around their true values. TL-kurtosis, $K R_{6}$, is severely biased in samples of size 50 , but then quickly converges to its true value. See Figure 6 . Table 7 illustrates that the bias of both $K R_{5}$ and $K R_{6}$ quickly vanishes as the sample size grows.

\subsubsection{Normal distribution with a single outlier}

Table $6(c, d)$ shows that for samples of 50 observations contaminating the standard Normal draws with a single outlier has noticeable effects on all measures, except $S K_{2}$ and $S K_{6}$. While $S K_{1}$ is severely affected by the introduction of single, very large, negative value even in samples of size $T=5000, S K_{3}, S K_{4}$ and $S K_{5}$ are mostly unaffected. In this case, in samples of 250 observations or more L-skewness delivers a RMSE ratio comparable with or, often lower than - that of either $S K_{2}$ and $S K_{3}$.

The effects of a single outlier on the conventional measure of kurtosis $K R_{1}$ are noteworthy: $K R_{1}$ becomes completely unreliable, even for $T=5000$. See Figure 5 . As expected, 
Table 6: Bias and RMSE ratio of skewness estimators - outliers

\begin{tabular}{ccccccc}
\hline$T$ & 50 & 250 & 500 & 1000 & 2500 & 5000 \\
\hline \multicolumn{7}{c}{$(a)$ Normal mixture } \\
\hline$S K_{1}$ & $2.147(1.00)$ & $1.532(1.00)$ & $1.402(1.00)$ & $0.816(1.00)$ & $0.447(1.00)$ & $0.199(1.00)$ \\
$S K_{2}$ & $-0.002(0.077)$ & $-0.001(0.032)$ & $-0.003(0.021)$ & $-0.003(0.016)$ & $0.001(0.012)$ & $0.001(0.011)$ \\
$S K_{3}$ & $0.004(0.057)$ & $-0.001(0.025)$ & $0.000(0.016)$ & $-0.001(0.013)$ & $0.001(0.010)$ & $0.001(0.008)$ \\
$S K_{4}$ & $0.005(0.043)$ & $0.001(0.019)$ & $0.001(0.012)$ & $-0.000(0.009)$ & $0.001(0.007)$ & $0.001(0.006)$ \\
$S K_{5}$ & $0.003(0.036)$ & $-0.001(0.018)$ & $0.002(0.011)$ & $0.000(0.009)$ & $0.000(0.007)$ & $0.000(0.006)$ \\
$S K_{6}$ & $-0.001(0.025)$ & $-0.001(0.010)$ & $-0.000(0.006)$ & $0.000(0.005)$ & $0.000(0.004)$ & $0.000(0.003)$ \\
\hline \multicolumn{7}{c}{$(b) \mathrm{N}(0,1)$ with 1987 Outlier } \\
\hline$S K_{1}$ & $-6.319(1.00)$ & $-11.722(1.00)$ & $-13.200(1.00)$ & $-12.654(1.00)$ & $-9.246(1.00)$ & $-5.984(1.00)$ \\
$S K_{2}$ & $-0.018(0.029)$ & $-0.002(0.007)$ & $-0.000(0.005)$ & $-0.001(0.003)$ & $-0.001(0.003)$ & $0.001(0.003)$ \\
$S K_{3}$ & $-0.449(0.073)$ & $-0.143(0.013)$ & $-0.076(0.007)$ & $-0.040(0.004)$ & $-0.017(0.003)$ & $-0.008(0.003)$ \\
$S K_{4}$ & $-0.135(0.022)$ & $-0.056(0.005)$ & $-0.035(0.003)$ & $-0.023(0.002)$ & $-0.011(0.002)$ & $-0.005(0.002)$ \\
$S K_{5}$ & $-0.522(0.083)$ & $-0.188(0.016)$ & $-0.104(0.008)$ & $-0.055(0.004)$ & $-0.023(0.003)$ & $-0.011(0.002)$ \\
$S K_{6}$ & $-0.009(0.009)$ & $-0.003(0.002)$ & $-0.001(0.001)$ & $-0.001(0.001)$ & $-0.000(0.001)$ & $0.000(0.001)$ \\
\hline \multicolumn{7}{c}{$(c) \mathrm{N}(0,1)$ with 2008 Outlier } \\
\hline$S K_{1}$ & $-4.422(1.00)$ & $-4.008(1.00)$ & $-2.862(1.00)$ & $-1.733(1.00)$ & $-0.798(1.00)$ & $-0.415(1.00)$ \\
$S K_{2}$ & $-0.019(0.041)$ & $-0.002(0.020)$ & $-0.000(0.022)$ & $-0.001(0.024)$ & $-0.001(0.033)$ & $0.001(0.046)$ \\
$S K_{3}$ & $-0.227(0.056)$ & $-0.056(0.019)$ & $-0.027(0.018)$ & $-0.015(0.019)$ & $-0.006(0.024)$ & $-0.002(0.032)$ \\
$S K_{4}$ & $-0.112(0.027)$ & $-0.037(0.013)$ & $-0.019(0.012)$ & $-0.011(0.014)$ & $-0.005(0.019)$ & $-0.002(0.025)$ \\
$S K_{5}$ & $-0.277(0.063)$ & $-0.074(0.019)$ & $-0.038(0.015)$ & $-0.020(0.014)$ & $-0.008(0.014)$ & $-0.004(0.017)$ \\
$S K_{6}$ & $-0.009(0.013)$ & $-0.003(0.006)$ & $-0.001(0.006)$ & $-0.001(0.007)$ & $-0.000(0.009)$ & $0.000(0.013)$ \\
\hline
\end{tabular}

Notes: see notes to Table 2 and details in Section 3.1.1.

a comparison of Table $7(c)$ and $7(d)$ illustrates that this detrimental impact is positively associated with the size of the outlier. With the exception of $K R_{2}$, all robust measures highly sensitive to the existence of a single outlier when $T=50$. See also Figure 6 . 
Figure 3: Sample distribution of $S K_{1}$
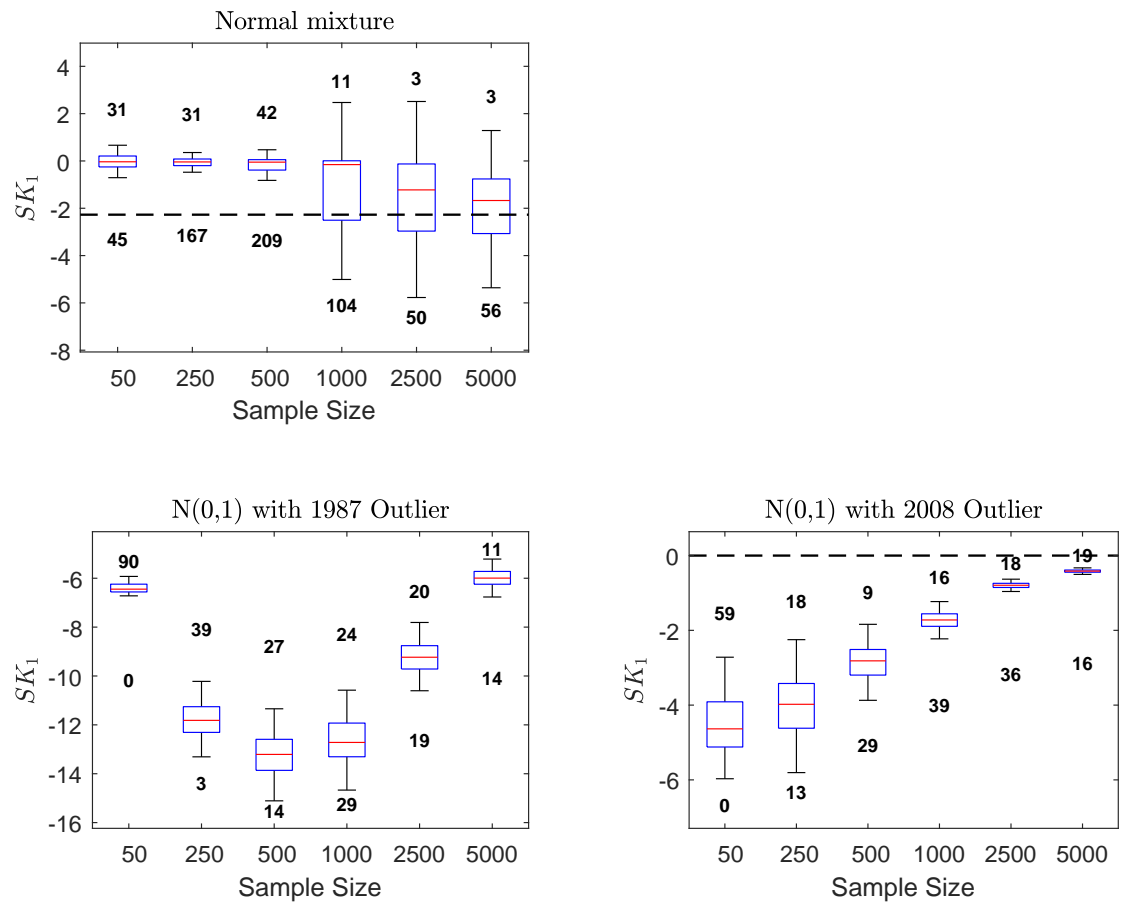

Notes: See notes to Figure 1 and details in Section 3.1.1.

Figure 4: Sample distribution of $S K_{6}$
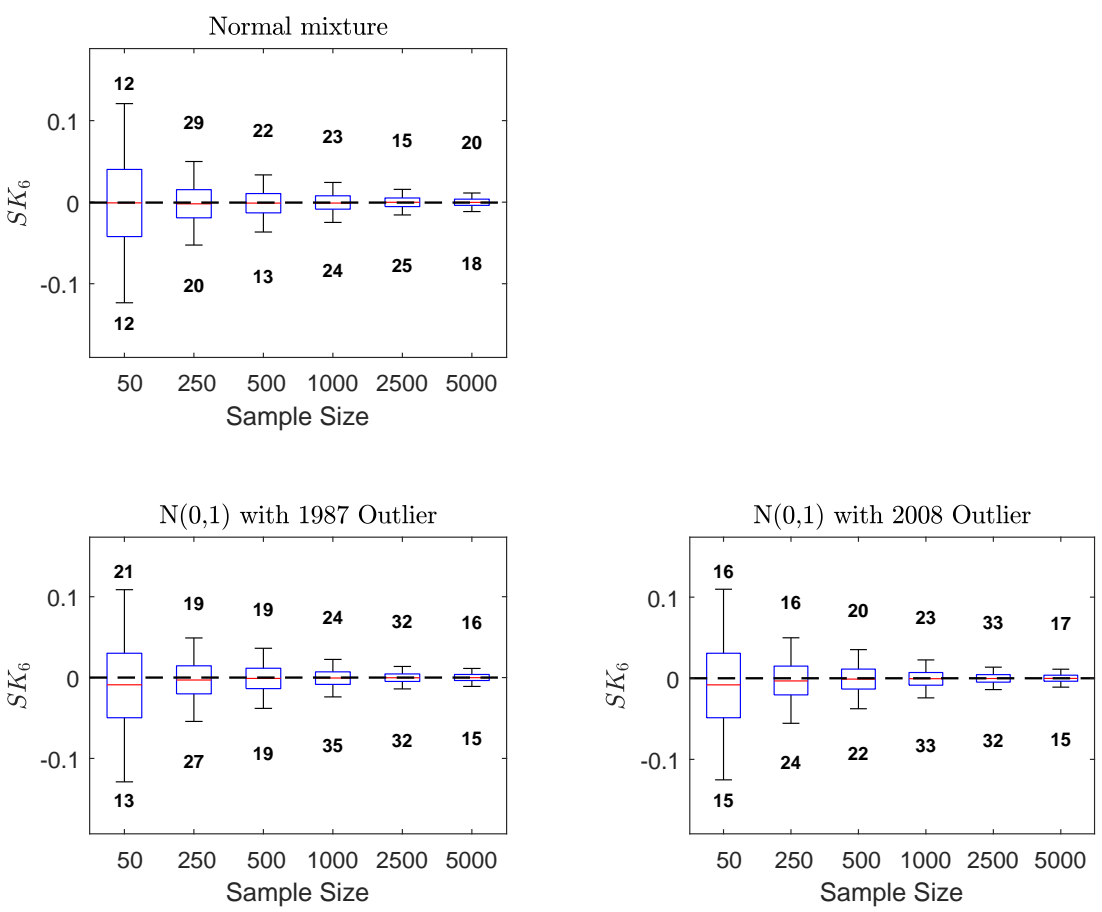

Notes: See notes to Figure 1 and details in Section 3.1.1. 
Table 7: Bias and RMSE ratio of excess kurtosis estimators - outliers

\begin{tabular}{lcccccc}
\hline$T$ & 50 & 250 & 500 & 1000 & 2500 & 5000 \\
\hline$K R_{1}$ & $-51.774(1.00)$ & $-43.619(1.00)$ & $-37.998(1.00)$ & $-26.370(1.00)$ & $-15.624(1.00)$ & $-7.918(1.00)$ \\
$K R_{2}$ & $0.026(0.005)$ & $0.007(0.002)$ & $0.000(0.002)$ & $0.001(0.001)$ & $0.001(0.001)$ & $0.001(0.001)$ \\
$K R_{3}$ & $-0.088(0.008)$ & $-0.013(0.005)$ & $-0.016(0.003)$ & $-0.036(0.002)$ & $-0.087(0.002)$ & $-0.128(0.003)$ \\
$K R_{4}$ & $0.326(0.039)$ & $-0.008(0.005)$ & $0.014(0.003)$ & $-0.002(0.002)$ & $0.003(0.002)$ & $0.003(0.001)$ \\
$K R_{5}$ & $-0.003(0.001)$ & $-0.001(0.001)$ & $-0.001(0.000)$ & $-0.000(0.000)$ & $0.000(0.000)$ & $0.000(0.000)$ \\
$K R_{6}$ & $-0.148(0.003)$ & $-0.010(0.000)$ & $-0.003(0.000)$ & $-0.001(0.000)$ & $0.000(0.000)$ & $0.000(0.000)$ \\
\hline \multicolumn{7}{c}{$(b) \mathrm{N}(0,1)$ with 1987 Outlier } \\
\hline$K R_{1}$ & $40.32(1.00)$ & $165.59(1.00)$ & $245.97(1.00)$ & $293.92(1.00)$ & $262.98(1.00)$ & $185.50(1.00)$ \\
$K R_{2}$ & $0.042(0.007)$ & $0.010(0.001)$ & $0.003(0.000)$ & $0.003(0.000)$ & $0.001(0.000)$ & $0.000(0.000)$ \\
$K R_{3}$ & $3.186(0.080)$ & $1.057(0.006)$ & $0.496(0.002)$ & $0.134(0.000)$ & $-0.100(0.000)$ & $-0.146(0.001)$ \\
$K R_{4}$ & $23.36(0.597)$ & $0.028(0.002)$ & $0.023(0.001)$ & $0.023(0.000)$ & $0.005(0.000)$ & $0.005(0.000)$ \\
$K R_{5}$ & $0.453(0.011)$ & $0.163(0.001)$ & $0.091(0.000)$ & $0.048(0.000)$ & $0.020(0.000)$ & $0.010(0.000)$ \\
$K R_{6}$ & $-0.145(0.004)$ & $-0.009(0.000)$ & $-0.002(0.000)$ & $-0.000(0.000)$ & $0.000(0.000)$ & $0.000(0.000)$ \\
\hline \multicolumn{7}{c}{$(c) \mathrm{N}(0,1)$ with 2008 Outlier } \\
\hline$K R_{1}$ & $24.977(1.00)$ & $39.550(1.00)$ & $31.900(1.00)$ & $20.611(1.00)$ & $9.964(1.00)$ & $5.231(1.00)$ \\
$K R_{2}$ & $0.038(0.010)$ & $0.009(0.003)$ & $0.003(0.002)$ & $0.002(0.003)$ & $0.000(0.004)$ & $0.000(0.005)$ \\
$K R_{3}$ & $1.492(0.059)$ & $0.381(0.010)$ & $0.175(0.006)$ & $0.051(0.003)$ & $-0.033(0.005)$ & $-0.051(0.011)$ \\
$K R_{4}$ & $7.772(0.313)$ & $0.028(0.006)$ & $0.023(0.005)$ & $0.022(0.006)$ & $0.004(0.008)$ & $0.005(0.010)$ \\
$K R_{5}$ & $0.236(0.009)$ & $0.062(0.002)$ & $0.033(0.001)$ & $0.017(0.001)$ & $0.007(0.001)$ & $0.004(0.001)$ \\
$K R_{6}$ & $-0.146(0.006)$ & $-0.009(0.000)$ & $-0.002(0.000)$ & $-0.000(0.000)$ & $0.000(0.000)$ & $0.000(0.001)$ \\
\hline
\end{tabular}

Notes: see notes to Table 2 and details in Section 3.1.1. 
Figure 5: Sample distribution of $K R_{1}$
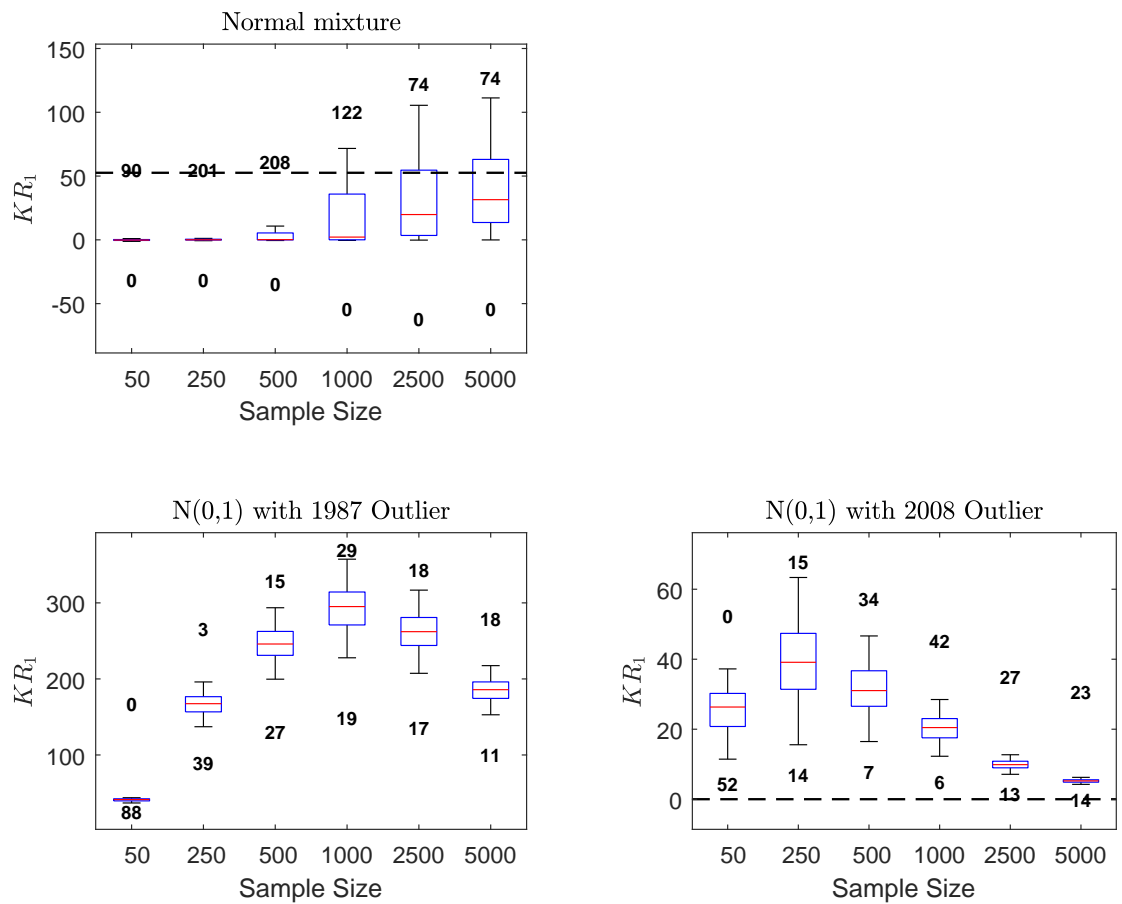

Notes: See notes to Figure 1 and details in Section 3.1.1.

Figure 6: Sample distribution of $K R_{6}$
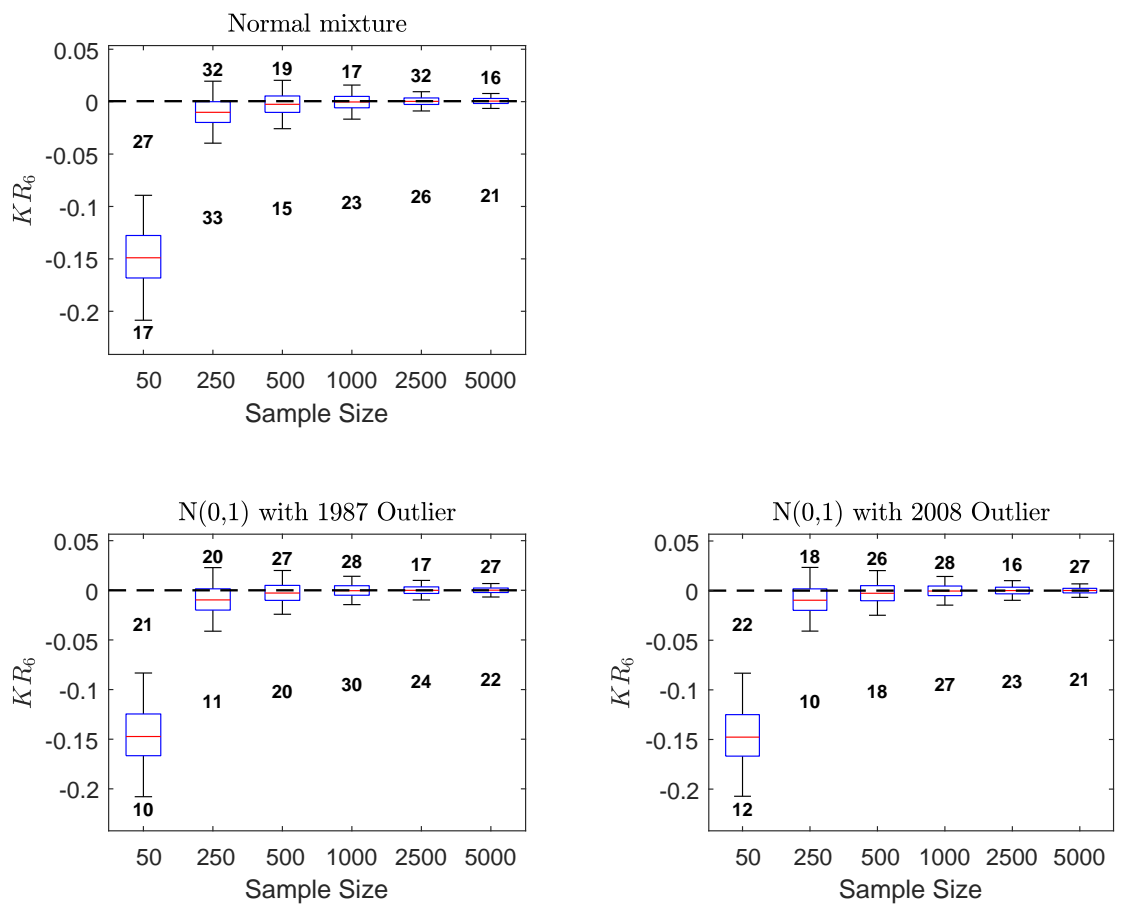

Notes: See notes to Figure 1 and details in Section 3.1.1. 


\section{Empirical application: the FRED-MD}

In this section we estimate the skewness and excess kurtosis of 128 US macroeconomic and financial time series in the FRED-MD (see McCracken and Ng, 2016, for details). This is a large, monthly frequency, dataset that focuses on the US economy and is updated in realtime through the FRED database. We rely on data for the period April 1959 - December 2018. Because of differences in data availability, the sample size ranges from 320 to 717 and is on average equal to 709. Transformations aimed at making the series stationary are applied when necessary. ${ }^{9}$

To gauge the maximum moment that exists for each series in the FRED-MD, we have estimated their maximal moment exponents (MME) relying on Hill (1975). The MME is defined as $\alpha_{\delta}=\sup _{q} E\left|x_{t}\right|^{q}<\infty$, where $q$ is the maximum exponent for which a finite moment exists. The MME can be estimated as follows (Hall, 1982):

$$
\hat{\alpha}_{\delta}=\left[\left(\frac{1}{m-1} \sum_{i=1}^{m-1} \log X_{i: T}\right)-\log X_{m: T}\right]^{-1} \quad \text { with } \delta=\frac{m}{T} \quad m>0
$$

we set $\delta=0.1$, so that we focus on the upper $10 \%$ of the empirical distribution of the series in the FRED-MD. ${ }^{10}$

Earlier empirical analyses showed that estimates of the MME for exchange rates and stock returns often lie between 2 and 4 (Chen et al., 2000; De Lima, 1997; Mittnik and Rachev, 1993; Loretan and Phillips, 1994); therefore, while the second moment seems to be finite, the third and fourth moments often are not. Our results are largely in line with these findings. In fact, Table 8 highlights that some of the series in the FRED-MD do not possess all moments required to compute conventional or even robust measures of skewness and kurtosis. The conventional skewness measure, that requires existence of moments up to order three, can be computed for 104 series out of 128. For the conventional kurtosis this number drops to 46 , meaning that only about $36 \%$ of the series possess moments up to the

\footnotetext{
${ }^{9}$ We apply the transformations suggested by McCracken and $\mathrm{Ng}$ (2016) to make the series stationary, but contrarily to them we do not remove outliers. In fact, we want to asses their impact of skewness and kurtosis. See the online Appendix to McCracken and Ng (2016) for details.

${ }^{10}$ It is well known that the Hill estimator is biased in small samples and is very sensitive to the choice of $\delta$ (see e.g. Kearns and Pagan, 1997; Huisman et al., 2001). For these reasons, we present some robustness checks in Section D.5 of the Appendix.
} 
fourth or higher. Therefore, these estimates suggest that there are 24 series $(19 \%$ of the total) that do not have finite third moment. Lastly, the estimated MME of the series in the FRED-MD exceeds 8 only in 15 out of 128 cases (11.7\% of the total).

All in all, there is evidence that at least some of the monthly US macroeconomic time series in the FRED-MD might not posses all moments that are necessary to conduct normality tests. For instance the Jarque-Bera procedure requires existence of the eighth moment, while a test of symmetry based on $S K_{1}$ requires existence of the sixth moment (Bai and $\mathrm{Ng}$, 2001). Problems associated with moment condition failure also arise when testing for nonlinearity (see e.g. Chen et al., 2000; De Lima, 1997).

For each estimator, Figure 7 presents a scatterplot that compares the skewness estimated on the whole sample and after deleting the largest observation in absolute value. Each point in Figure 7 refers to the estimate of the skewness for a different series in the FRED-MD. We can see that $S K_{2}$ and $S K_{6}$ display the lowest dispersion and a high degree of correlation, thus suggesting that they are the most robust measures. The same exercise for kurtosis is presented in Figure 8. The correlation between the estimated kurtosis for the whole sample and the "leave-one-out" estimate is highest for $K R_{6}^{\prime}$. Moreover, Figures 7 and 8 highlight that the conventional measures of skewness and kurtosis display the highest dispersion, thus confirming their low resistance to outlying observations.

\subsection{Selected series}

Following McCracken and $\mathrm{Ng}$ (2016) the 128 series in the FRED-MD can be assigned to eight groups: "Output \& Income" (16 series), "Labor Market" (31 series), "Housing" (10 series), "Consumption, Orders \& Inventories" (10 series), "Money \& Credit" (14 series), "Interest \& Exchange Rates" (22 series), "Prices" (20 series), "Stock Market" (5 series). We select one representative series in each group and in Table 8 we report the estimate of its MME, $S K_{1}$, $S K_{5}, K R_{1}$ and $K R_{5}$. We rely on measures based on L-moments because their performance is comparable with that of estimators based on TL-moments, but do not require to arbitrarily choose the number of observations to trim. Column 3 shows that, with exception of Housing Starts, none of the series has finite fourth moment. Notice that the lowest MME, 1.58, is 
Figure 7: Robustness of skewness measures for the FRED-MD
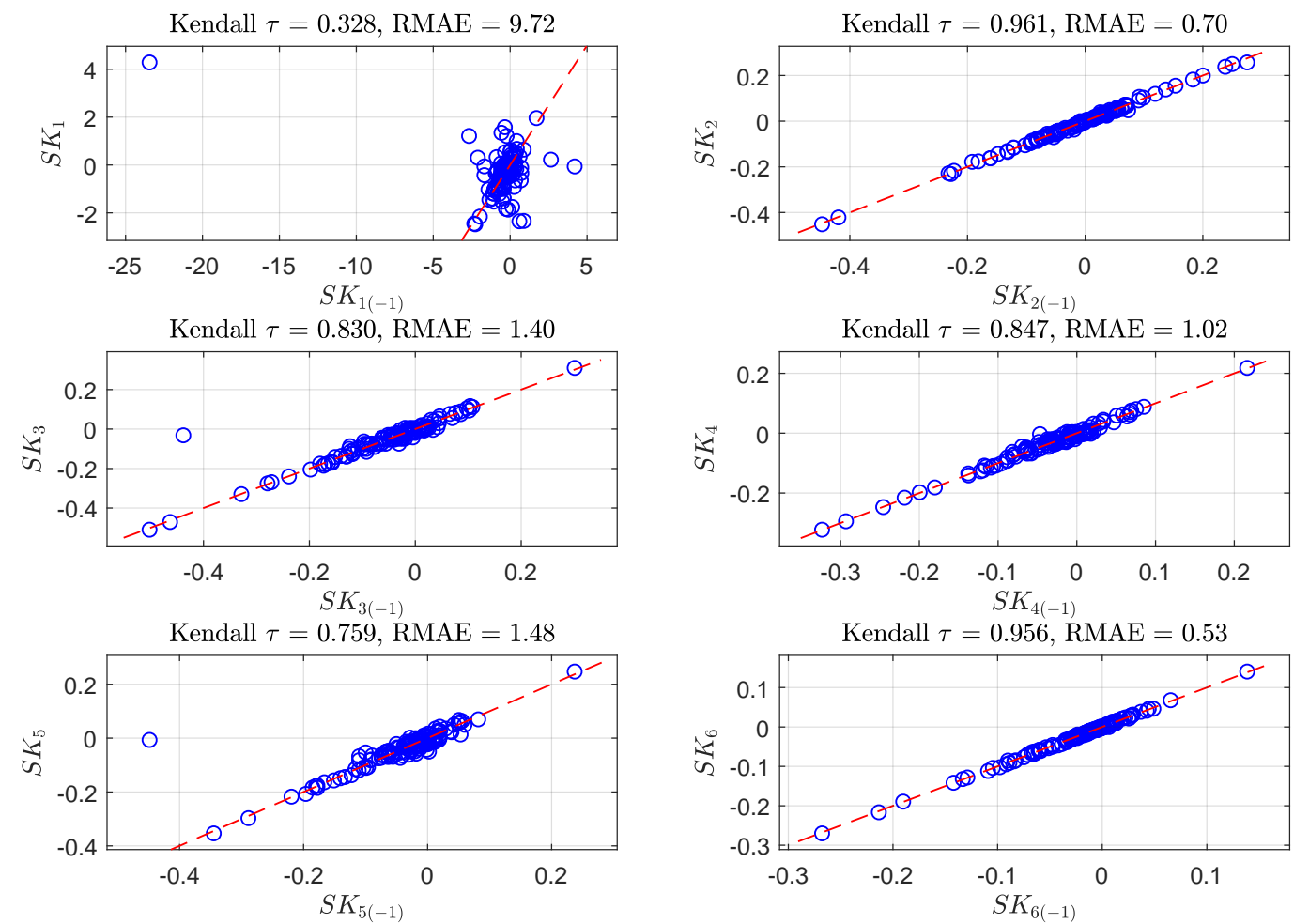

Notes: the figure shows the estimated skewness for each series in the FRED-MD (circles) with (x-axis) and without (y-axis) the largest observation (in absolute value). The dashed line is the $45^{\circ}$ line. Each figure also shows the Kemdall's $\tau$ correlation coefficient and the root mean absolute error (RMAE).

associated with the Fed Fund rate that also displays the largest excess kurtosis as measured both by $K R_{1}$ and $K R_{5}$. Comparing columns 6 and 7 , we can see that $K R_{1}$ is particularly sensitive to outliers. For instance, $K R_{1}$ for log-returns on the S\&P500 index is 4 if computed on the whole sample, while it halves when omitting the largest observation is absolute value. This sensitivity to outliers is also a feature of $S K_{1}$. On the contrary, $S K_{5}$ and $K R_{5}$ remain largely unaffected when one outlying observation is deleted. 
Figure 8: Robustness of kurtosis measures for the FRED-MD
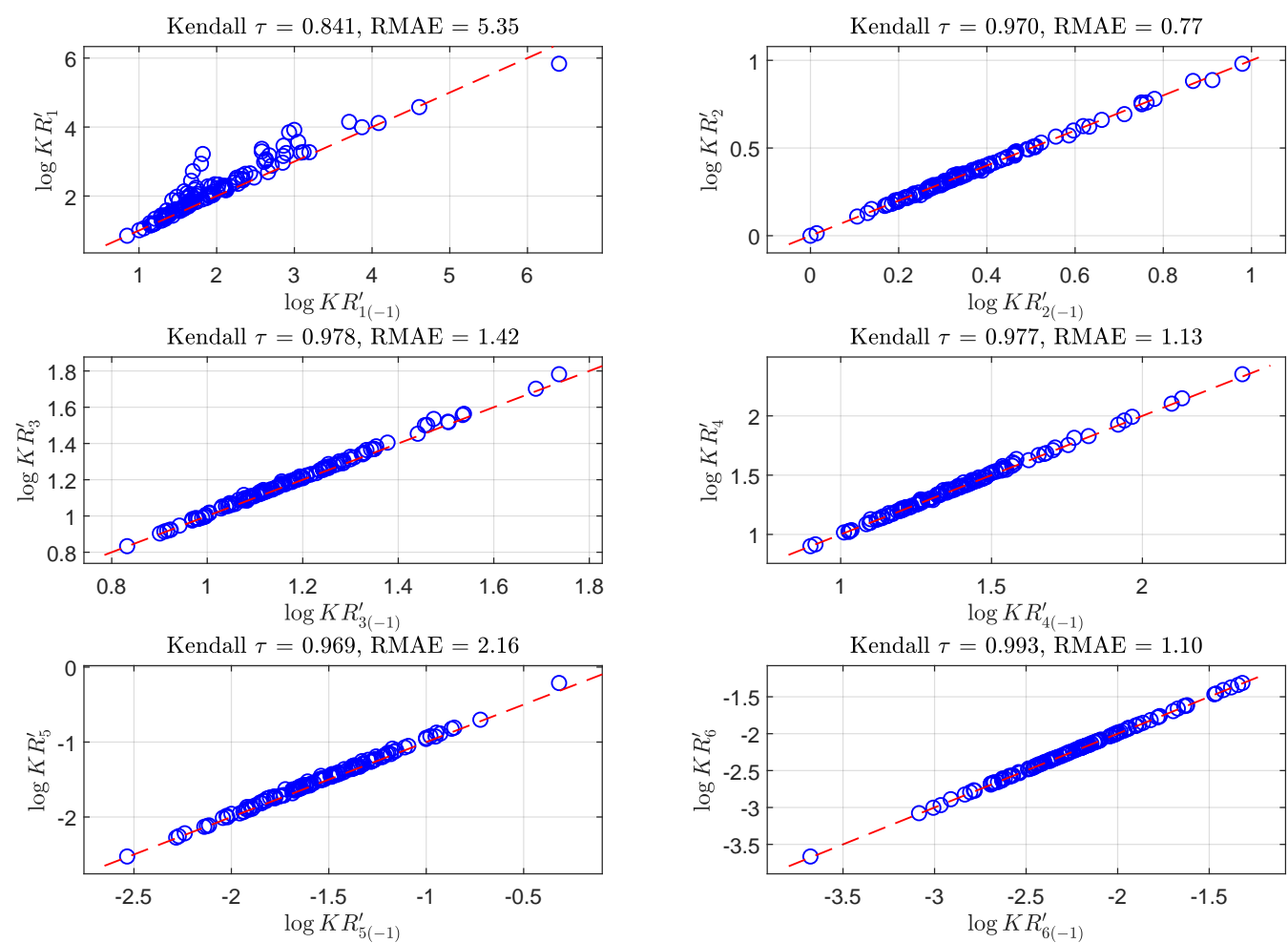

Notes: the figure shows the logarithm of the estimated kurtosis for each series in the FRED-MD (circles) with (x-axis) and without (y-axis) the largest observation (in absolute value). The dashed line is the $45^{\circ}$ line. See notes to Figure 7.

Table 8: Robustness of skewness and kurtosis for selected macroeconomic series in the FREDMD.

\begin{tabular}{lcccccccccc}
\hline Series & Trans. & $\hat{\alpha}_{10 \%}$ & $S K_{1}$ & $S K_{1(-1)}$ & $K R_{1}$ & $K R_{1(-1)}$ & $S K_{5}$ & $S K_{5(-1)}$ & $K R_{5}$ & $K R_{5(-1)}$ \\
\hline IP & $\Delta \log x_{t}$ & 2.898 & -0.344 & -0.940 & 7.319 & 4.555 & -0.051 & -0.068 & 0.102 & 0.091 \\
Un. Rate & $\Delta x_{t}$ & 2.153 & 0.512 & 0.373 & 1.786 & 1.177 & 0.064 & 0.055 & 0.070 & 0.064 \\
House & $\log x_{t}$ & 94.533 & -1.012 & -1.021 & 1.185 & 1.192 & -0.176 & -0.178 & 0.063 & 0.062 \\
Real PCE & $\Delta \log x_{t}$ & 3.075 & -0.205 & -0.006 & 2.875 & 2.078 & -0.011 & -0.001 & 0.088 & 0.081 \\
M2 & $\Delta^{2} \log x_{t}$ & 2.353 & -0.864 & -0.424 & 8.805 & 6.446 & -0.043 & -0.028 & 0.145 & 0.135 \\
Fed Funds & $\Delta x_{t}$ & 1.588 & -2.340 & 0.884 & 47.247 & 17.065 & -0.043 & -0.001 & 0.323 & 0.302 \\
CPI & $\Delta^{2} \log x_{t}$ & 2.165 & 0.015 & -0.402 & 4.843 & 2.814 & -0.014 & -0.028 & 0.104 & 0.095 \\
S\&P 500 & $\Delta \log x_{t}$ & 3.263 & -1.031 & -0.713 & 4.184 & 2.095 & -0.108 & -0.096 & 0.081 & 0.072 \\
\hline
\end{tabular}

Notes: column 1 reads as follows: IP = Industrial Production; Un. Rate = Unemployment Rate; House = Housing Starts; Real PCE $=$ Real personal consumption expenditures; M2 = M2 Money Stock; Fed Funds = Effective Federal Funds Rate; CPI $=$ CPI All Items; S\&P500 = S\&P 500 Index. Column 2 shows the transformation applied to make the series stationary (see McCracken and Ng, 2016); column 3 reports point estimates of maximal moment exponent; columns 4-11 show skewness and excess kurtosis measures computed on the whole sample $\left(S K_{i}, K R_{i}\right)$ or leaving out the largest observation (in absolute value) of the series $\left(S K_{i,(-1)}, K R_{i,(-1)}\right)$. The full sample has size 717 for all series. 


\section{Conclusions}

In this paper we have assessed the relative merits of different estimators of skewness and excess kurtosis. Our Monte Carlo analysis highlights that standard estimators based on the method-of-moments feature a higher bias than more robust and easily implementable alternatives. This result is not confined to data generating processes contaminated with outlying observations, but carries over to non-Gaussian samples and even to normally distributed random variables featuring serial correlation and persistence.

In the empirical application to the FRED-MD we provide further evidence that methodof-moments estimators of skewness and kurtosis are unreliable for macroeconomic time series. Moreover, we also show that several time series do not possess enough finite moments to perform standard test of Normality, symmetry or nonlinearity (Ramsey and Rothman, 1996). Since these tests are often used not only for model-building purposes, but also to investigate substantive questions in economics and finance (Bontemps and Meddahi, 2005; Kilian and Demiroglu, 2000) our results have far reaching implications for empirical analyses based on macroeconomic and financial time series. These are not limited to estimation of skewness and kurtosis, but possibly extend to testing normality and symmetry (Bai and Ng, 2001).

On a more positive note, we show that robust estimators are available and perform much better - both in term of bias reduction and lower dispersion - than standard measures of skewness and kurtosis. In particular, our main contribution is to introduce L- and TL-moments in the time series econometrics literature illustrating that these estimators outperform those based on the method-of-moments, as well as alternative robust estimators based on quantiles. Moreover, L- and TL-moments can be used to characterize a very large class of statistical distributions because they are less demanding in terms the moments. On disadvantage of TL-moments is that they involve to arbitrarily select the degree of trimming. All in all our results open the doors for the use of L and TL-moments in macroeconomic and financial applications, where there is often an issue of moment failure (Chen et al., 2000; De Lima, 1997; Mittnik and Rachev, 1993; Loretan and Phillips, 1994). Two potential applications of L- and TL-moments, that we leave for future investigation, are tests of normality and symmetry in the time series domain and the estimation and forecasting of 
realized volatility.

\section{References}

Bai, J. and Ng, S. (2001). A consistent test for conditional symmetry in time series models. Journal of Econometrics, 103(1-2):225-258.

Bai, J. and Ng, S. (2005). Tests for skewness, kurtosis, and normality for time series data. Journal of Business $\&$ Economic Statistics, 23(1):49-60.

Bollerslev, T. (1987). A conditionally heteroskedastic time series model for speculative prices and rates of return. Review of economics and statistics, 69(3):542-547.

Bonato, M. (2011). Robust estimation of skewness and kurtosis in distributions with infinite higher moments. Finance Research Letters, 8(2):77-87.

Bontemps, C. and Meddahi, N. (2005). Testing normality: a GMM approach. Journal of Econometrics, 124(1):149-186.

Bowley, A. L. (1920). Elements of Statistics. P.S. King \& Sons.

Brys, G., Hubert, M., and Struyf, A. (2003). A comparison of some new measures of skewness. In Dutter, R., Filzmoser, P., Gather, U., and Rousseeuw, P. J., editors, Developments in Robust Statistics, pages 98-113. Heidelberg: Springer-Verlag.

Brys, G., Hubert, M., and Struyf, A. (2004). A robust measure of skewness. Journal of Computational and Graphical Statistics, 13(4):996-1017.

Cecchetti, S. G., Lam, P.-S., and Mark, N. C. (1990). Mean reversion in equilibrium asset prices. American Economic Review, 80(3):398-418.

Chen, Y.-T., Chou, R. Y., and Kuan, C.-M. (2000). Testing time reversibility without moment restrictions. Journal of Econometrics, 95(1):199-218.

Crow, E. L. and Siddiqui, M. (1967). Robust estimation of location. Journal of the American Statistical Association, 62(318):353-389. 
Darolles, S., Gouriéroux, C., and Jasiak, J. (2009). L-performance with an application to hedge funds. Journal of Empirical Finance, 16(4):671-685.

David, H. A. and Nagaraja, H. N. (2017). Order Statistics. Wiley.

De Lima, P. J. (1997). On the robustness of nonlinearity tests to moment condition failure. Journal of Econometrics, 76(1-2):251-280.

DeLong, J. B. and Summers, L. H. (1986). Are business cycles symmetrical. In Gordon, R. J., editor, The American Business Cycle: Continuity and Change, pages 166-178. University of Chicago Press.

Denby, L. and Martin, R. D. (1979). Robust estimation of the first-order autoregressive parameter. Journal of the American Statistical Association, 74(365):140-146.

Elamir, E. A. and Seheult, A. H. (2003). Trimmed L-moments. Computational Statistics \&6 Data Analysis, 43(3):299-314.

Engle, R. F. and Gallo, G. M. (2006). A multiple indicators model for volatility using intra-daily data. Journal of Econometrics, 131(1-2):3-27.

Franses, P. H. and Van Dijk, D. (2000). Non-linear time series models in empirical finance. Cambridge University Press.

Gleason, J. R. (1993). Understanding elongation: The scale contaminated normal family. Journal of the American Statistical Association, 88(421):327-337.

Groeneveld, R. A. and Meeden, G. (1984). Measuring skewness and kurtosis. Journal of the Royal Statistical Society. Series D, 33(4):391-399.

Hall, P. (1982). On some simple estimates of an exponent of regular variation. Journal of the Royal Statistical Society. Series B, 44(1):37-42.

Harri, A. and Coble, K. H. (2011). Normality testing: two new tests using L-moments. Journal of Applied Statistics, 38(7):1369-1379. 
Hill, B. M. (1975). A simple general approach to inference about the tail of a distribution. The Annals of Statistics, 3(5):1163-1174.

Hinkley, D. V. (1975). On power transformations to symmetry. Biometrika, 62(1):101-111.

Hogg, R. V. (1972). More light on the kurtosis and related statistics. Journal of the American Statistical Association, 67(338):422-424.

Hogg, R. V. (1974). Adaptive robust procedures: A partial review and some suggestions for future applications and theory. Journal of the American Statistical Association, 69(348):909-923.

Hosking, J. (1992). Moments or L moments? An example comparing two measures of distributional shape. The American Statistician, 46(3):186-189.

Hosking, J. (2007). Some theory and practical uses of trimmed L-moments. Journal of Statistical Planning and Inference, 137(9):3024-3039.

Hosking, J. R. (1990). L-moments: analysis and estimation of distributions using linear combinations of order statistics. Journal of the Royal Statistical Society. Series B, pages $105-124$.

Hubert, M. and Debruyne, M. (2009). Breakdown value. Wiley International Reviews Computational Statistics, 1:296-302.

Huisman, R., Koedijk, K. G., Kool, C. J. M., and Palm, F. (2001). Tail-index estimates in small samples. Journal of Business \& Economic Statistics, 19(2):208-216.

Karvanen, J. (2006). Estimation of quantile mixtures via l-moments and trimmed l-moments. Computational Statistics \& Data Analysis, 51(2):947-959.

Kearns, P. and Pagan, A. (1997). Estimating the density tail index for financial time series. Review of Economics and Statistics, 79(2):171-175.

Kilian, L. and Demiroglu, U. (2000). Residual-based tests for normality in autoregressions: asymptotic theory and simulation evidence. Journal of Business 8 Economic Statistics, $18(1): 40-50$. 
Kim, T.-H. and White, H. (2004). On more robust estimation of skewness and kurtosis. Finance Research Letters, 1(1):56-73.

Loretan, M. and Phillips, P. C. B. (1994). Testing the covariance stationarity of heavy-tailed time series: an overview of the theory with applications to several financial datasets. Journal of Empirical Finance, 1(2):211-248.

McCracken, M. W. and Ng, S. (2016). FRED-MD: A monthly database for macroeconomic research. Journal of Business \&5 Economic Statistics, 34(4):574-589.

McCracken, M. W. and Ng, S. (2016). FRED-MD: A monthly database for macroeconomic research. Journal of Business EF Economic Statistics, 34(4):574-589.

Meijer, E. (2000). An asymmetric distribution with zero skewness. Technical report, Available at SSRN: http://dx.doi.org/10.2139/ssrn.2531847.

Mittnik, S. and Rachev, S. T. (1993). Modeling asset returns with alternative stable distributions. Econometric Reviews, 12(3):261-330.

Moors, J. J. A. (1988). A quantile alternative for kurtosis. Journal of the Royal Statistical Society. Series D, 37(1):25-32.

Ord, J. K. (1968). The discrete Student's $t$ distribution. The Annals of Mathematical Statistics, 39(5):1513-1516.

Ramsey, J. B. and Rothman, P. (1996). Time irreversibility and business cycle asymmetry. Journal of Money, Credit and Banking, 28(1):1-21.

Scott, R. C. and Horvath, P. A. (1980). On the direction of preference for moments of higher order than the variance. Journal of Finance, 35(4):915-919.

Thomas, G. (2009). Assessing symmetry using quantiles and L-moments. Communications in Statistics- Simulation and Computation, 38(2):335-354.

von Hippel, P. (2010). Skewness. In Lovric, M., editor, International Encyclopedia of Statistical Science. Springer. 
Westfall, P. H. (2014). Kurtosis as peakedness, 1905-2014. RIP. The American Statistician, 68(3):191-195.

Wooldridge, J. M. (2012). Introductory Econometrics: A Modern Approach. South-Western, 5th edition. 


\section{Appendix to "Robust measures of skewness and kurtosis for financial and macroeconomic time series"}

\section{A Monte Carlo design: further details}

\section{A.1 Standard Normal distributions contaminated with a single outlier}

To produce the two Monte Carlo designs in which we contaminate the standard Normal distribution with a single outlier, we follow Kim and White (2004) and rely on the sample distribution of daily log-returns on the S\&P500 index. We downloaded adjusted close prices from Yahoo! Finance. We consider two sample periods: 04/01/1982-06/05/1999 and 29/06/2001-30/11/2018. In both cases the size of the sample is 4384. For each period, we identify the minimum return that corresponds to $-22.90 \%$ in $19 / 10 / 1987$ (first sample) and to $-9.47 \%$ in 15/10/2008 (second sample). Outliers are injected in the simulated distributions at time $\tau_{i}$ for $i=1,2$, that corresponds to the location of the outlier in the sample divided by the total number of observations. This approach leads to $\tau_{1}=0.30$ and $\tau_{2}=0.48$. The size of outliers, $m_{i}$ for $i=1,2$ is determined as: $m_{i}=x_{\left\lfloor\tau_{i} T\right\rfloor} / Q_{0.25}$. This yields: $m_{1}=-22.90 /-0.45=50.89$ and $m_{2}=-9.47 /-0.49=19.33$. The last step is as follows: in each of the two experiment, we simulate of a sample of size $T$ from the standard Normal distribution, we calculate $Q_{0.25}$ and then we replace $x_{\left\lfloor\tau_{i} T\right\rfloor}$ with the outlier $m_{i} \times Q_{0.25}$. 


\section{B Estimation of sample skewness \& kurtosis}

\section{B.1 Conventional skewness and kurtosis}

Given a sample of size $T,\{x\}_{t=1}^{T}, S K_{1}$ and $K R_{1}$ are estimated as follows:

$$
\begin{aligned}
& \widehat{S K}_{1}=\frac{1}{T} \sum_{t=1}^{T}\left(\frac{x_{t}-\hat{\mu}}{\hat{\sigma}}\right)^{3} \\
& \widehat{K R}_{1}=\frac{1}{T} \sum_{t=1}^{T}\left(\frac{x_{t}-\hat{\mu}}{\hat{\sigma}}\right)^{4}-3
\end{aligned}
$$

where $\hat{\mu}=T^{-1} \sum_{t=1}^{T} x_{t}$ and $\hat{\sigma}=\sqrt{T^{-1} \sum_{t=1}^{T}\left(x_{t}-\hat{\mu}\right)^{2}}$.

\section{L-moments \& TL moments: further details}

\section{C.1 Estimation of L-moments}

L-moments can be generally defined as:

$$
\lambda_{r}=\frac{1}{r} \sum_{j=0}^{r-1}(-1)^{j}\left(\begin{array}{c}
r-1 \\
j
\end{array}\right) E\left(X_{r-j: r}\right) \quad \text { for } r=1,2, \ldots
$$

The expectation of an order statistics, $E\left(X_{j: r}\right)$, can be written as (David and Nagaraja, 2017):

$$
\begin{aligned}
E\left(X_{j: r}\right) & =\frac{r !}{(j-1) !(r-j) !} \int_{-\infty}^{\infty} x F^{j-1}(x)[1-F(x)]^{r-j} f(x) \mathrm{d} x \\
& =\frac{r !}{(j-1) !(r-j) !} \int_{0}^{1} u^{j-1}[1-u]^{r-j} Q(u) \mathrm{d} u
\end{aligned}
$$

Substituting (3) in (2) and rearranging, we get:

$$
\lambda_{r}=\int_{0}^{1} P_{r-1}^{*}(u) Q(u) \mathrm{d} u
$$

where $P_{r}^{*}(u)=\sum_{j=0}^{r} p_{r, j}^{*} u^{j}$ and $p_{r, j}^{*}=(-1)^{r-j}\left(\begin{array}{c}r \\ j\end{array}\right)\left(\begin{array}{c}r+j \\ j\end{array}\right)$. Notice that $P_{r}^{*}(u)$ is the $r$-th shifted Legendre polynomial, related to the Legendre polynomial by $P_{r}^{*}(u)=P_{r}(2 u-1)$. 
L-moments can be estimated by sample L-moments. These are defined as:

$$
\ell_{r}=\sum_{j=0}^{r-1} p_{r-1, j}^{*} b_{j}
$$

where:

$$
b_{j}=\frac{1}{n} \sum_{i=1}^{n} \frac{(i-1)(i-2) \ldots(i-j)}{(n-1)(n-2) \ldots(n-j)} x_{i: n}
$$

\section{C.2 TL-moments: existence, bounds and estimation}

The general definition of TL-moment is as follows:

$$
\lambda_{r}^{(s, k)}=\frac{1}{r} \sum_{j=0}^{r-1}(-1)^{j}\left(\begin{array}{c}
r-1 \\
j
\end{array}\right) E\left(X_{r+s-j: r+s+k}\right)
$$

Hosking (2007) provided a sufficient condition for the existence of TL-moments that are showed to exist whenever $E\left\{\left[\max \left(-X_{t}, 0\right)^{1} /(s+1)\right]\right\}<\infty$ and $E\left\{\left[\max \left(X_{t}, 0\right)^{1} /(k+1)\right]\right\}<$ $\infty$

In the empirical analysis we set $s=k=1$, therefore the first four TL-moments can be written as: ${ }^{11}$

$$
\begin{aligned}
\lambda_{1}^{(1,1)} & =E\left(X_{2: 3}\right)=6 \int_{0}^{1} Q(u) u(1-u) \mathrm{d} u \\
\lambda_{2}^{(1,1)} & =\frac{1}{2} E\left(X_{3: 4}-X_{2: 4}\right)=6 \int_{0}^{1} Q(u) u(1-u)(2 u-1) \mathrm{d} u \\
\lambda_{3}^{(1,1)} & =\frac{1}{3} E\left(X_{4: 5}-2 X_{3: 5}+X_{2: 5}\right)=\frac{20}{3} \int_{0}^{1} Q(u) u(1-u)\left(5 u^{2}-5 u+1\right) \mathrm{d} u \\
\lambda_{4}^{(1,1)} & =\frac{1}{4} E\left(X_{5: 6}-3 X_{4: 6}+3 X_{3: 6}-X_{2: 6}\right)=\ldots \\
& =\frac{15}{2} \int_{0}^{1} Q(u) u(1-u)\left(14 u^{3}-21 u^{2}+9 u-1\right) \mathrm{d} u
\end{aligned}
$$

$\lambda_{1}^{(1,1)}$ and $\lambda_{2}^{(1,1)}$ are thus robust measures of location and scale. With $s=k=1$ the condition for the existence of TL-moments is $E\left(\left|X_{t}\right|^{0.5}\right)<\infty$. For example, since the mean of the Cauchy distribution the cannot be defined, its L-moments cannot be defined either. However,

\footnotetext{
${ }^{11}$ In this case, with $k=s=1$, the $r$-th TL-moment is defined in terms of a conceptual sample of size equal to $r+s+k=r+2$.
} 
since $E\left(\left|X_{t}\right|^{0.5}\right)=1.41$ its TL-moment, $\lambda_{r}^{(1,1)}$, does exist. ${ }^{12}$

Bounds on TL moment ratios. Hosking (2007) showed that for the generic TL-moment ratio $\tau_{r}^{(s, k)} \equiv \lambda_{r}^{(r, s)} / \lambda_{2}^{(r, s)} \forall r \geq 3$ a bound can be defined as follows:

$$
\left|\tau_{r}^{(s, k)}\right| \leq \frac{2(m+1) !(r+s+k) !}{r(m+r-1) !(2+s+k) !} \quad \text { where } m=\min (s, k)
$$

for $s=k=0$ we get $\left|\tau_{r}\right| \leq r ! / r(r-1)$ ! that simplifies to $\left|\tau_{r}\right|<1 \quad \forall r \geq 3$. For $s=k=1$ the bound on TL-moment ratios is: $\left|\tau_{r}^{(1,1)}\right| \leq(1 / 6)(r+2) ! /(r \times r !)$. Therefore, we get: $\left|\tau_{3}^{(1,1)}\right|=\left|S K_{6}\right| \leq 1.11$ and $\left|\tau_{4}^{(1,1)}\right|=\left|K R_{6}^{\prime}\right| \leq 1.25$.

Sample TL moments. TL-moments can be consistently and unbiasedly estimated as:

$$
\ell_{r}^{(s, k)}=\frac{1}{r\left(\begin{array}{c}
n \\
t+s+k
\end{array}\right)} \sum_{j=s+1}^{n-k} \sum_{i=0}^{r-1}(-1)^{i}\left(\begin{array}{c}
r-1 \\
i
\end{array}\right)\left(\begin{array}{c}
j-1 \\
r+s-i-1
\end{array}\right)\left(\begin{array}{c}
n-j \\
k+i
\end{array}\right) x_{j}
$$

\footnotetext{
${ }^{12}$ We used Matlab's numerical integration routine "integral" to solve $E\left(\left|X_{t}\right|^{0.5}\right)=\int_{-\infty}^{\infty}\left|X_{t}\right|^{0.5} f\left(X_{t}\right) \mathrm{d} X_{t}$ assuming that $X_{t}$ has a standard Cauchy distribution with probability density function $f\left(X_{t}\right)=$ $1 /\left[\pi\left(1+X_{t}^{2}\right)\right]$.
} 


\section{Additional tables \& figures}

D.1 Additional figures - Statistical Distributions 
Figure A1: Statistical distributions used in Monte Carlo simulations
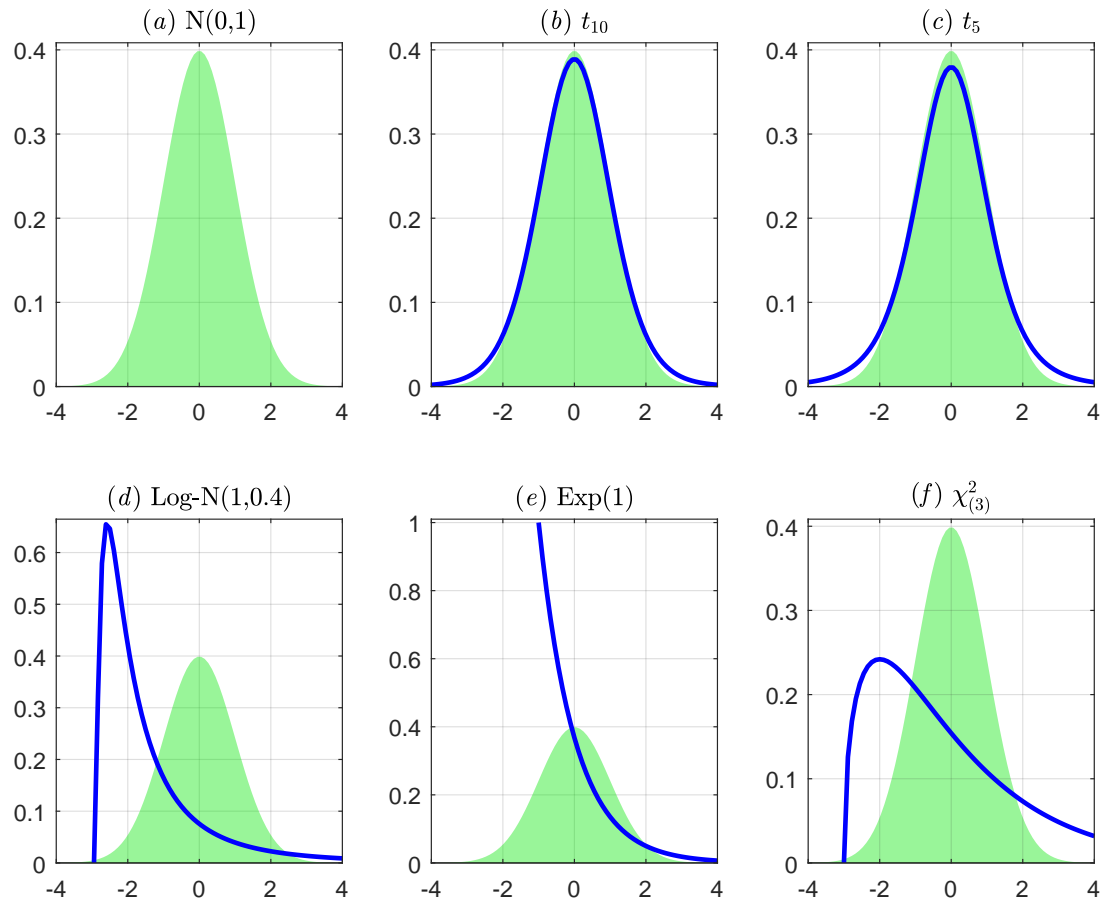

Notes: each panel shows the probability density function (pdf) of the standard Normal distribution as a filled area. Thick continuous lines represent the pdf for the distribution indicated in the title of each panel. Distributions in panel $(d-f)$ have been centered to have zero mean. 
Figure A2: Normal Mixture and standard Normal with a single outlier
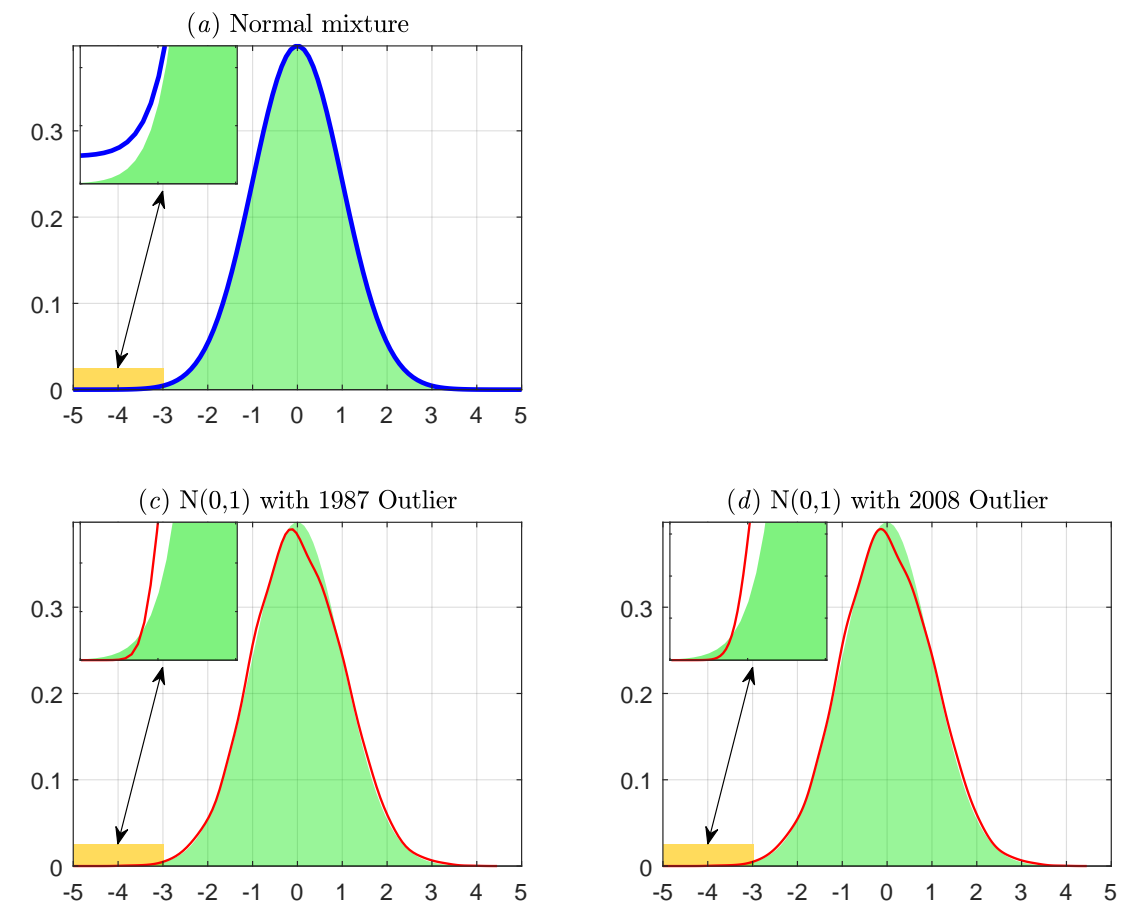

Notes: each panel shows the probability density function (pdf) of the standard Normal distribution as a filled area. Thick continuous lines represent the pdf for the distribution indicated in the title of each panel. Thin continuous lines in panel $(c)$ and $(d)$ represent the kernel estimate of the pdf of the standard Normal distribution contaminated with a single outlier. Subfigures in panel $(a, c, d)$ show the magnification of the left tail of the distribution. See Section A.1 of the Appendix for details on distributions (c) and (d). 
D.2 Additional figures - iid samples 
Figure A3: Sample distribution of $S K_{2}$
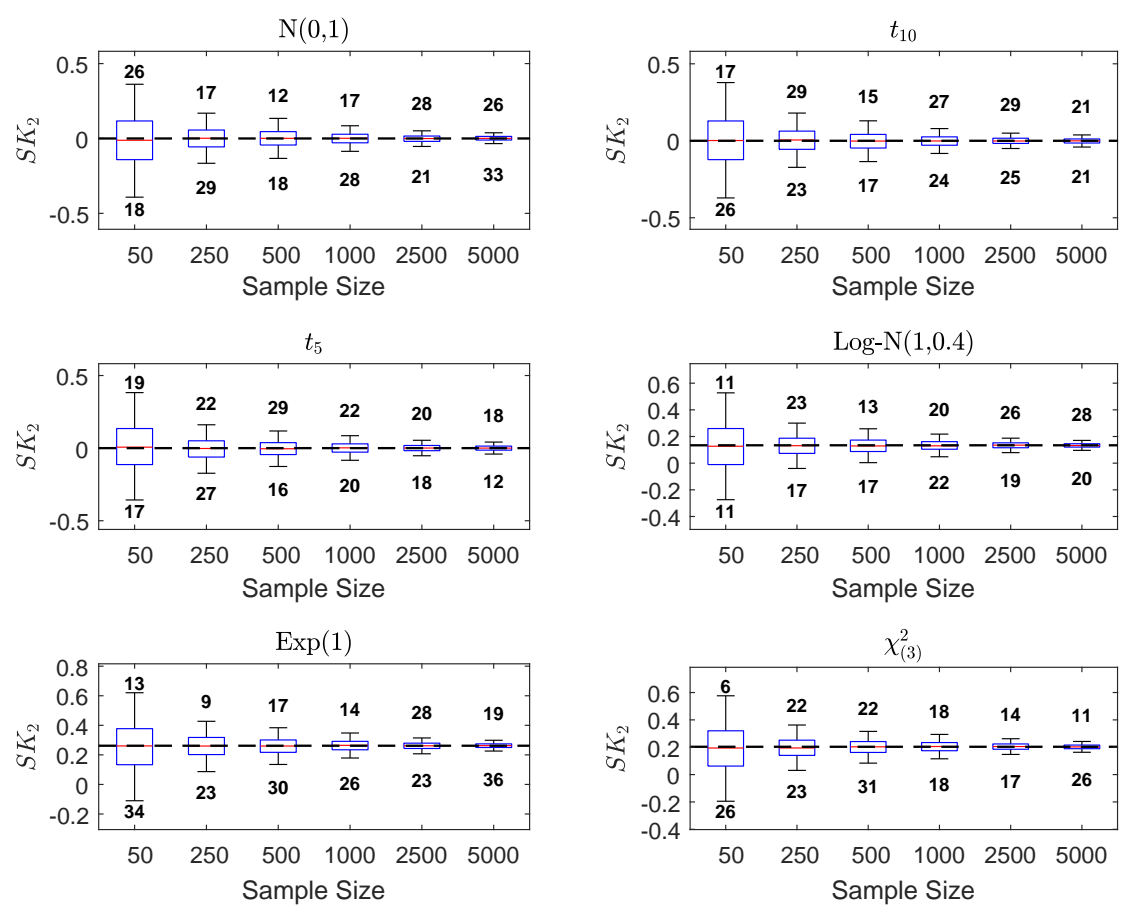

Notes: See notes to Figure 1.

Figure A4: Sample distribution of $S K_{3}$
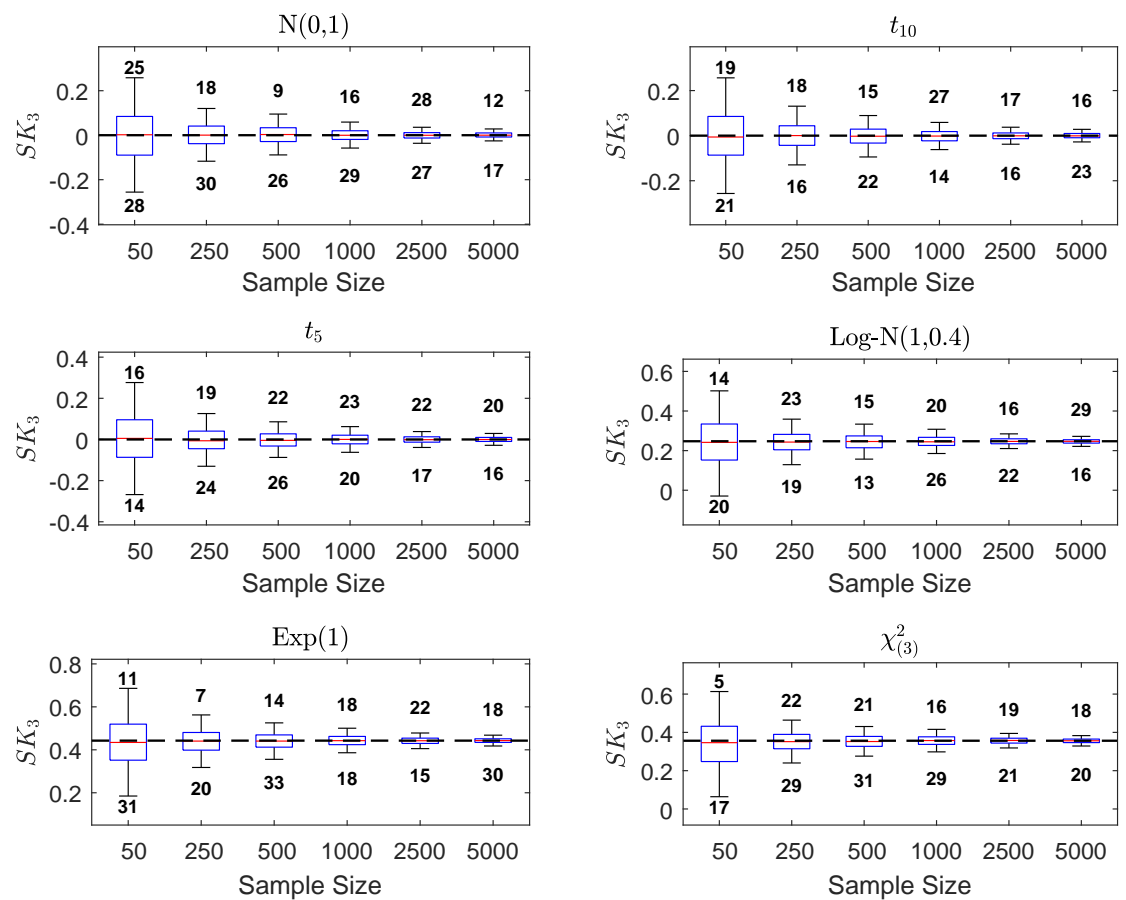

Notes: See notes to Figure 1. 
Figure A5: Sample distribution of $\mathrm{SK}_{4}$
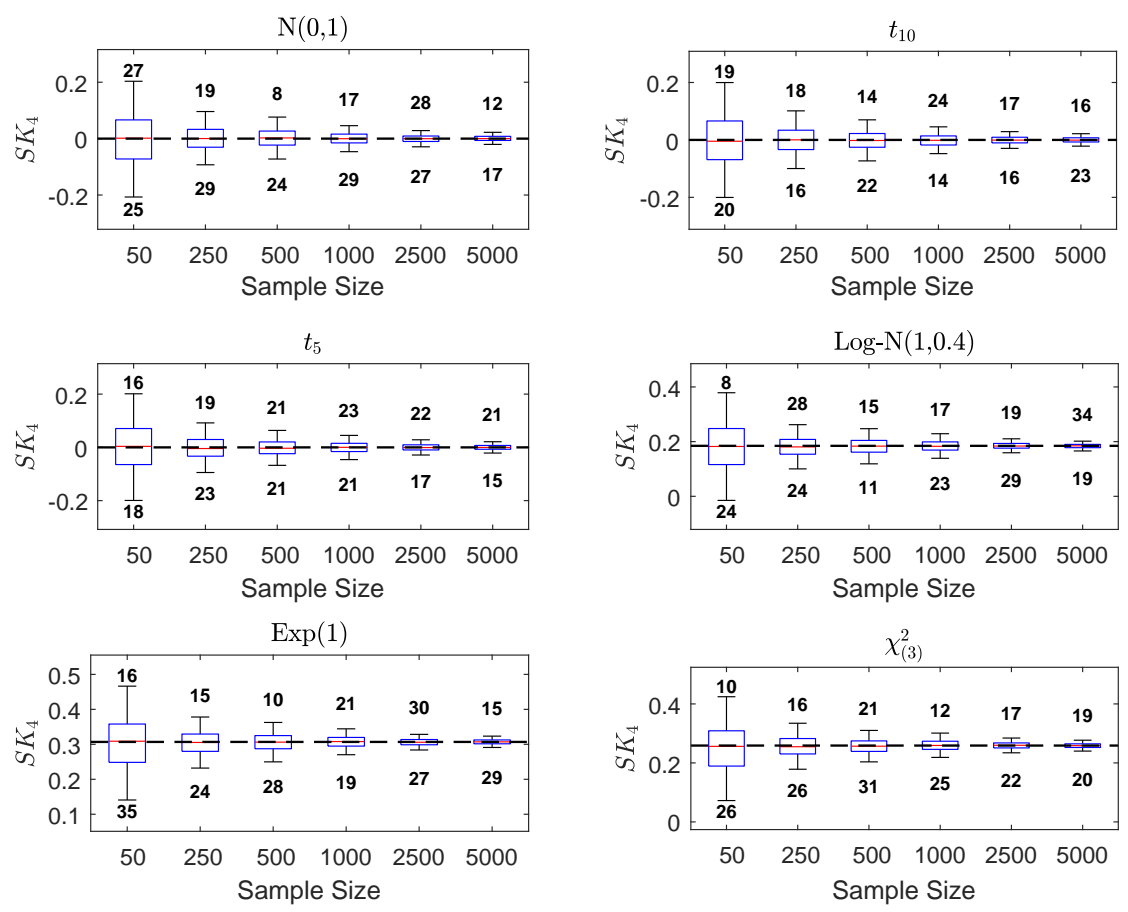

Notes: See notes to Figure 1.

Figure A6: Sample distribution of $S K_{5}$
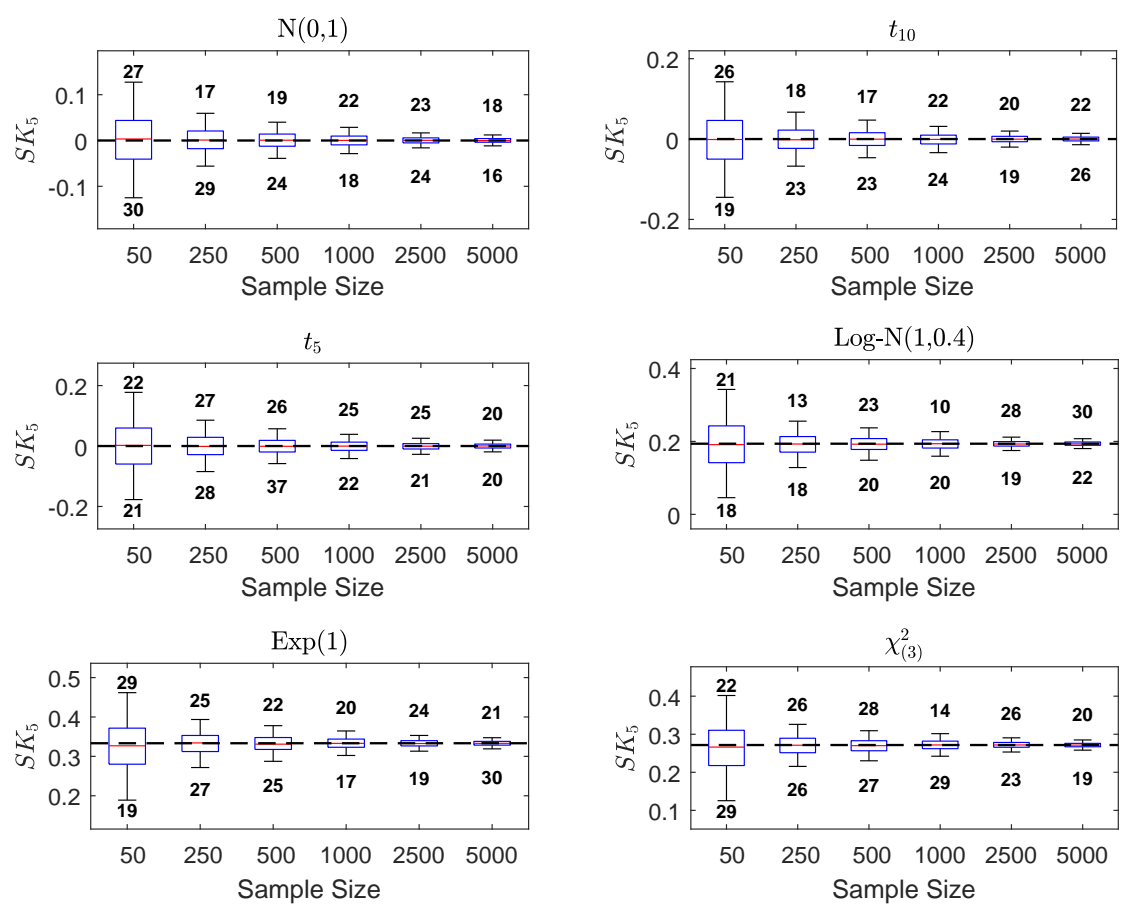

Notes: See notes to Figure 1. 
Figure A7: Sample distribution of $S K_{6}$
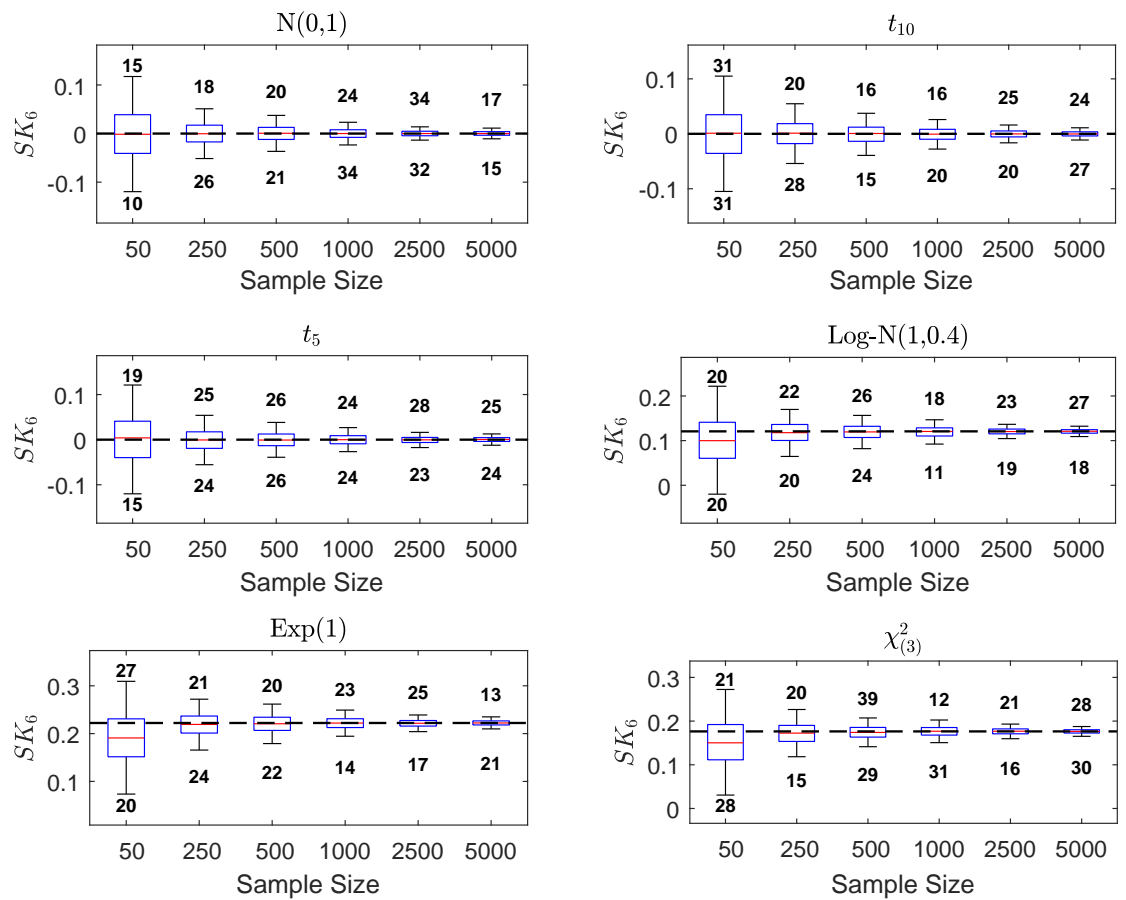

Notes: See notes to Figure 1. 
Figure A8: Sample distribution of $K R_{2}$
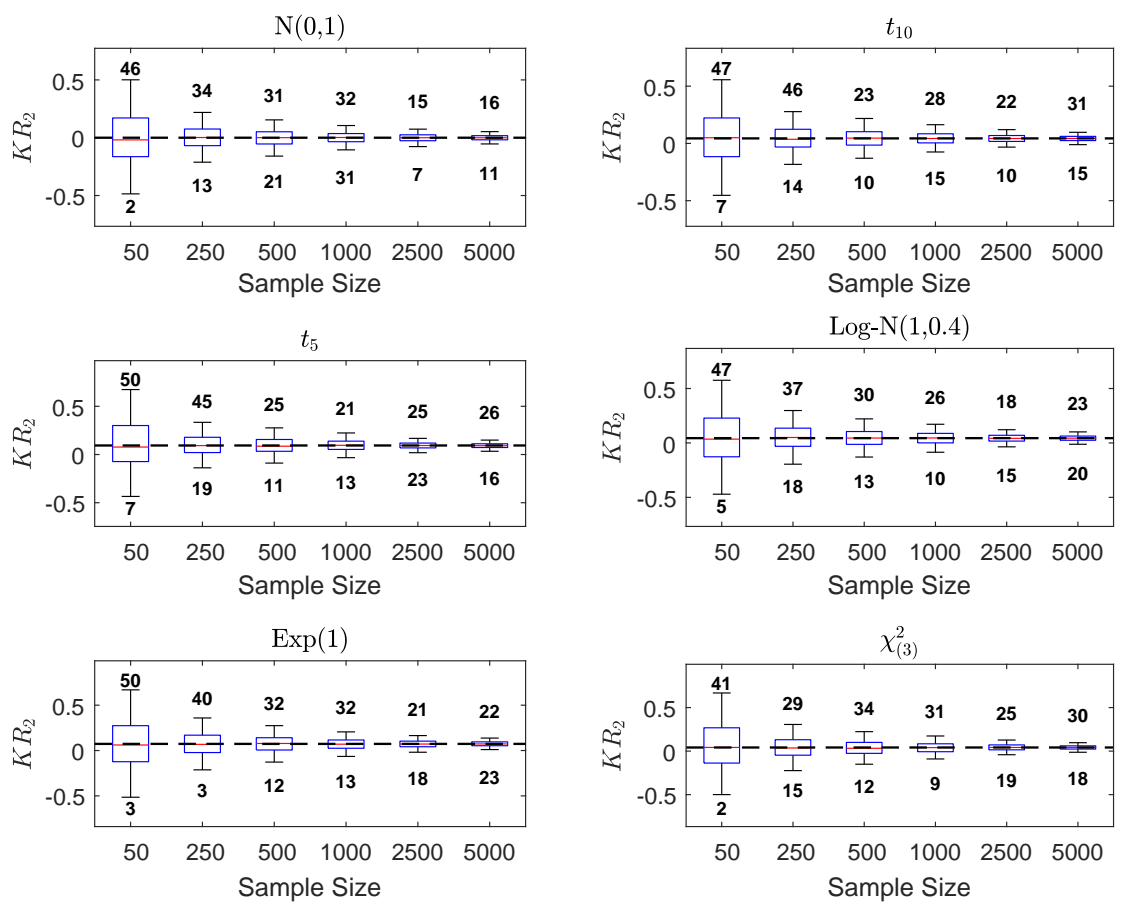

Notes: See notes to Figure 1.

Figure A9: Sample distribution of $K R_{3}$
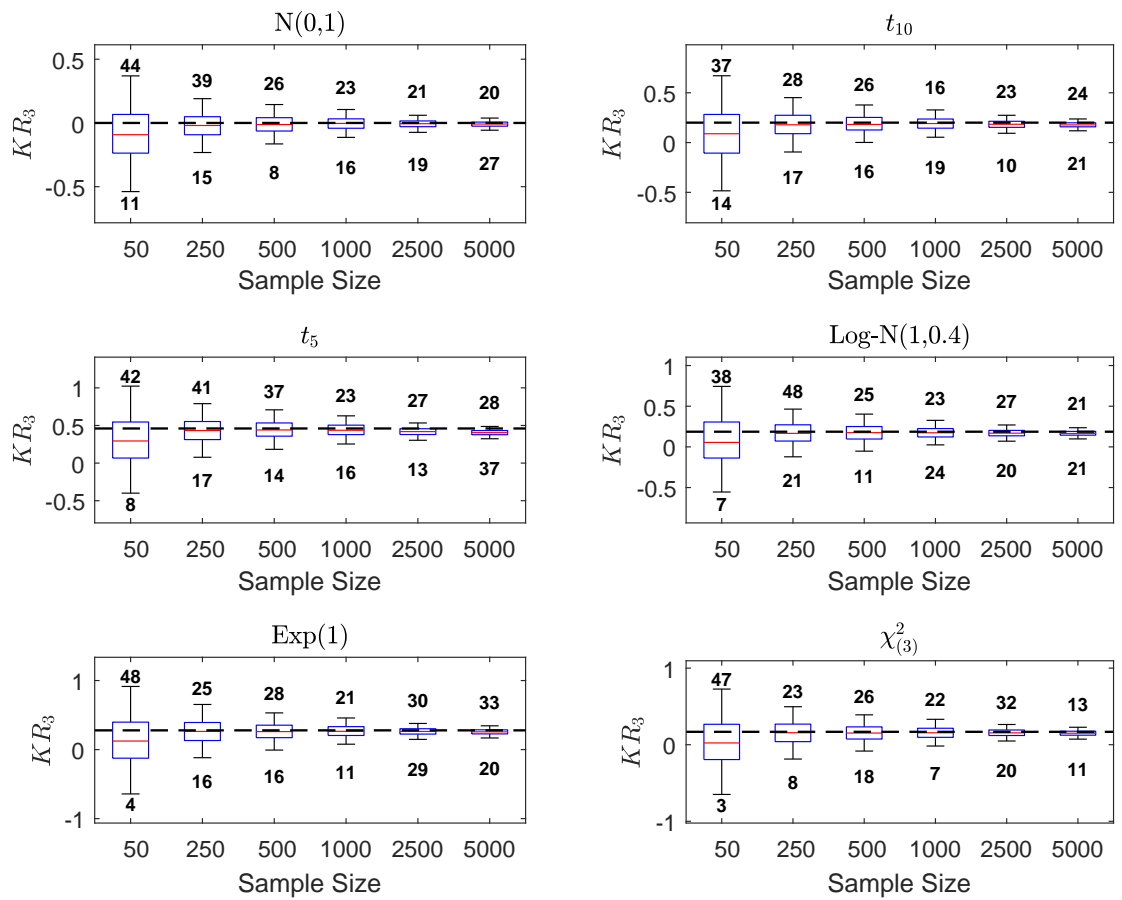

Notes: See notes to Figure 1. 
Figure A10: Sample distribution of $K R_{4}$
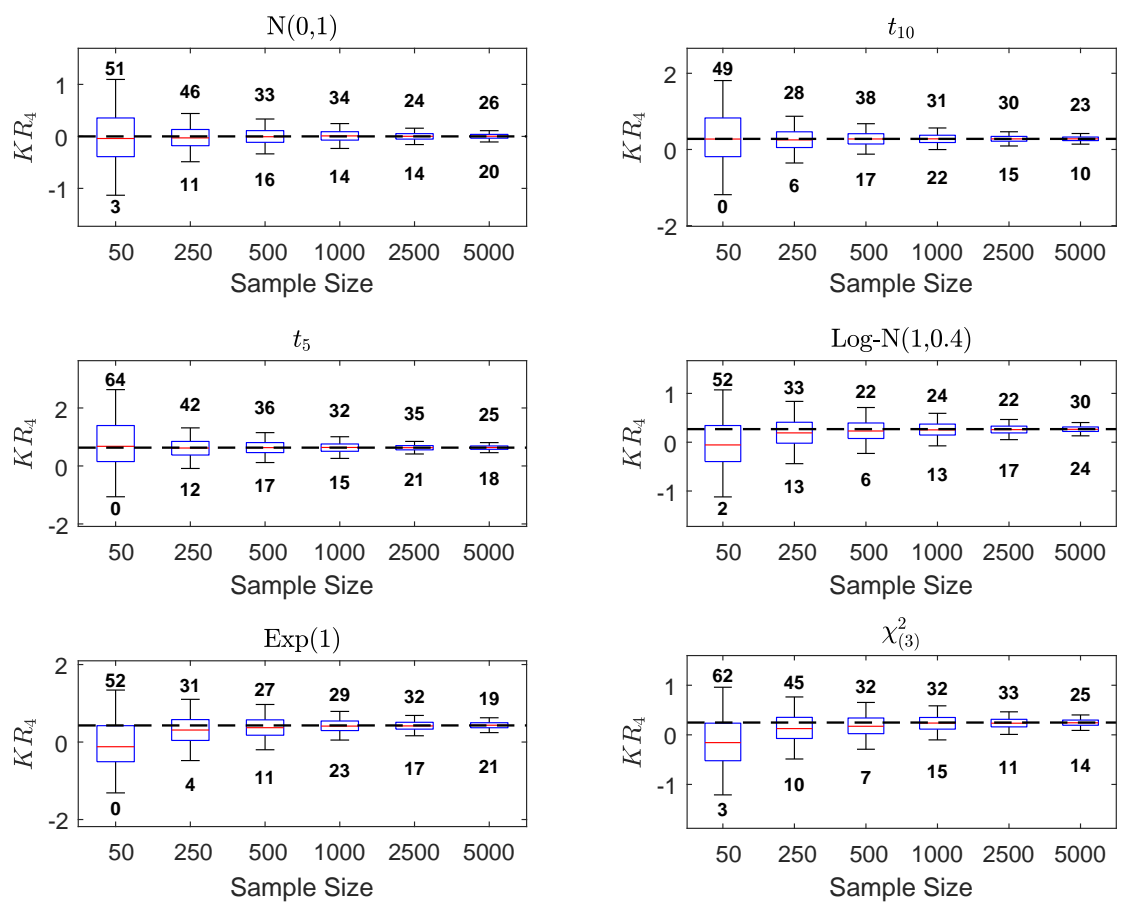

Notes: See notes to Figure 1.

Figure A11: Sample distribution of $K R_{5}$
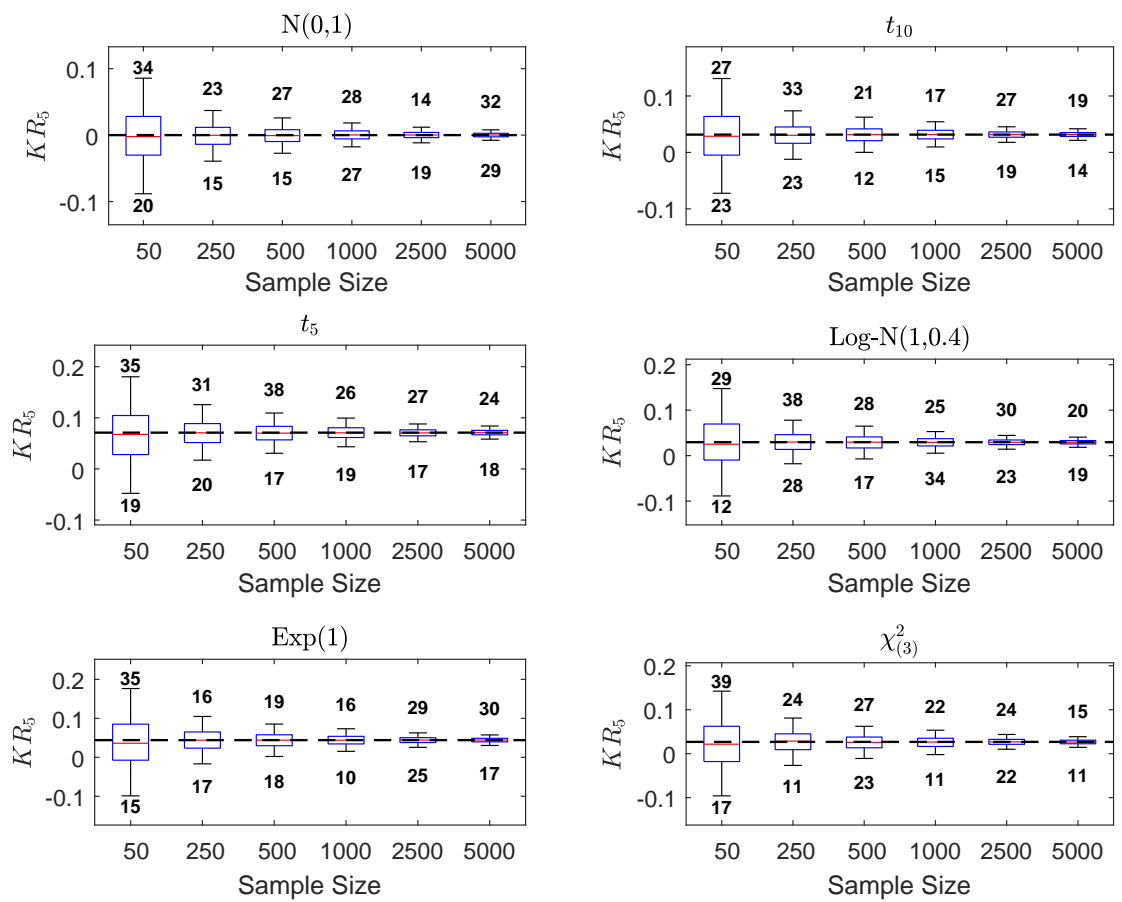

Notes: See notes to Figure 1. 
Figure A12: Sample distribution of $K R_{6}$
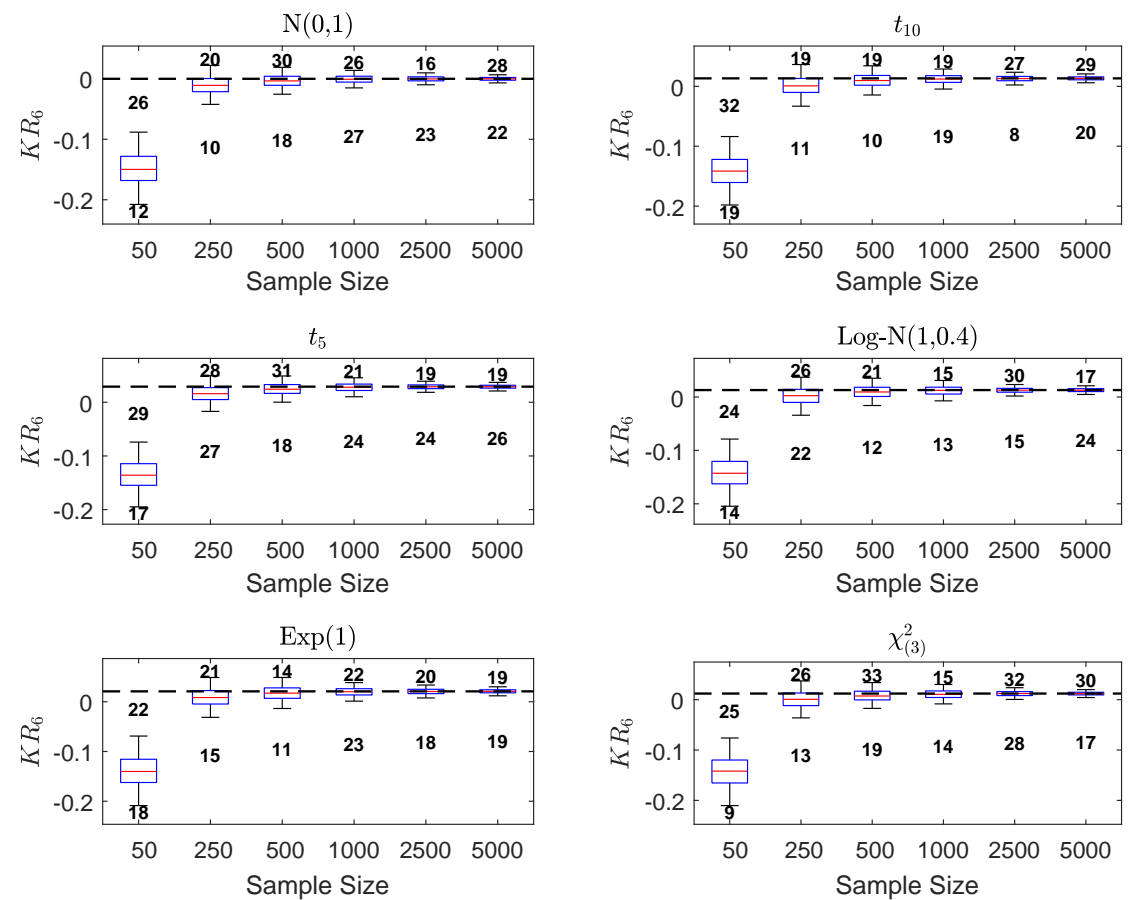

Notes: See notes to Figure 1. 
D.3 Additional figures - outliers 
Figure A13: Sample distribution of $S K_{2}$
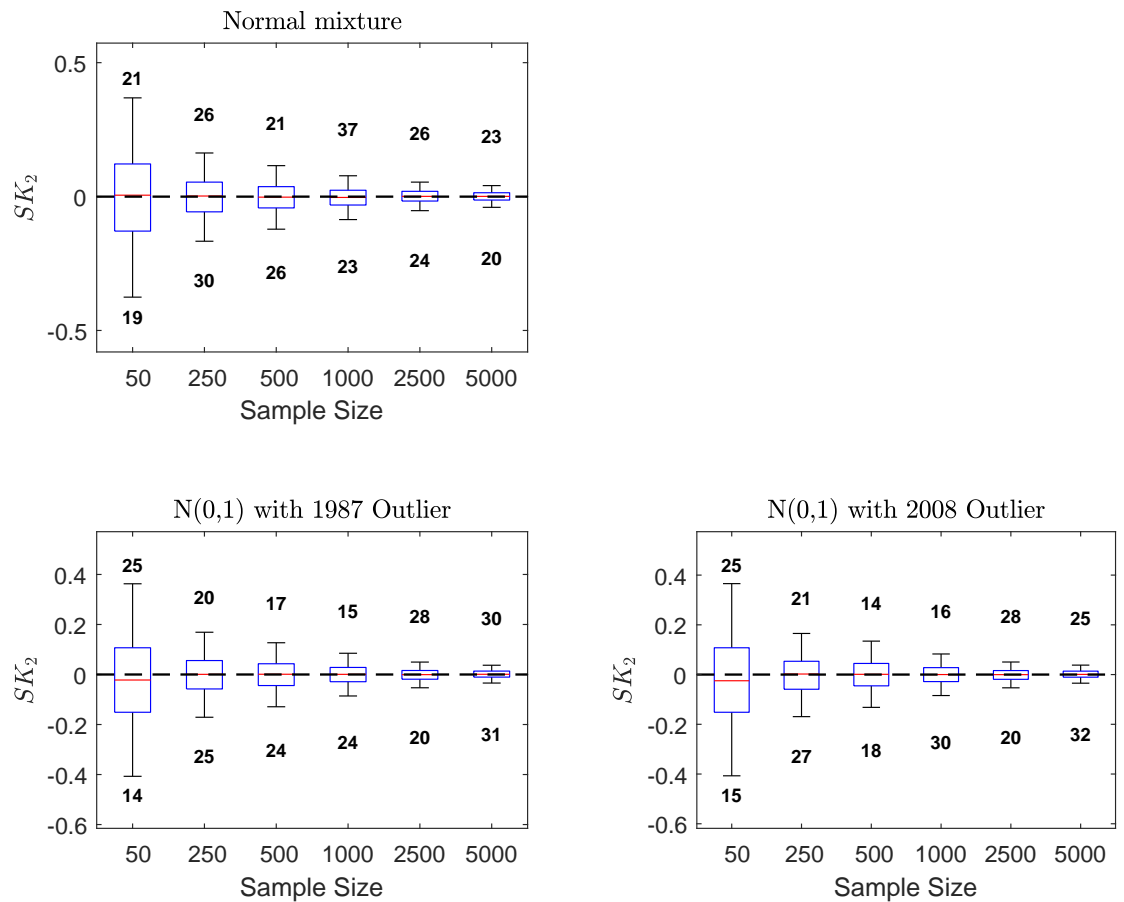

Notes: See notes to Figure 1 and details in Section 3.1.1.

Figure A14: Sample distribution of $S K_{3}$
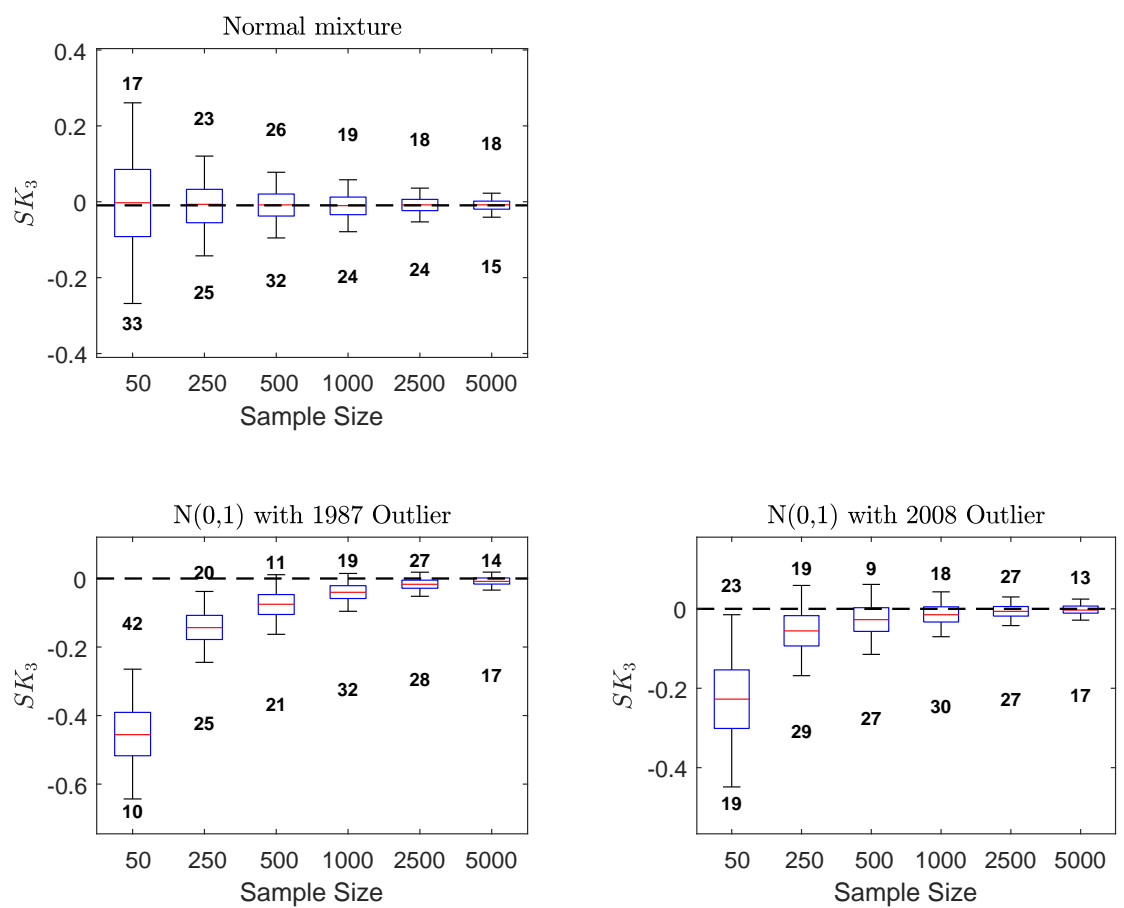

Notes: See notes to Figure 1 and details in Section 3.1.1. 
Figure A15: Sample distribution of $S K_{4}$
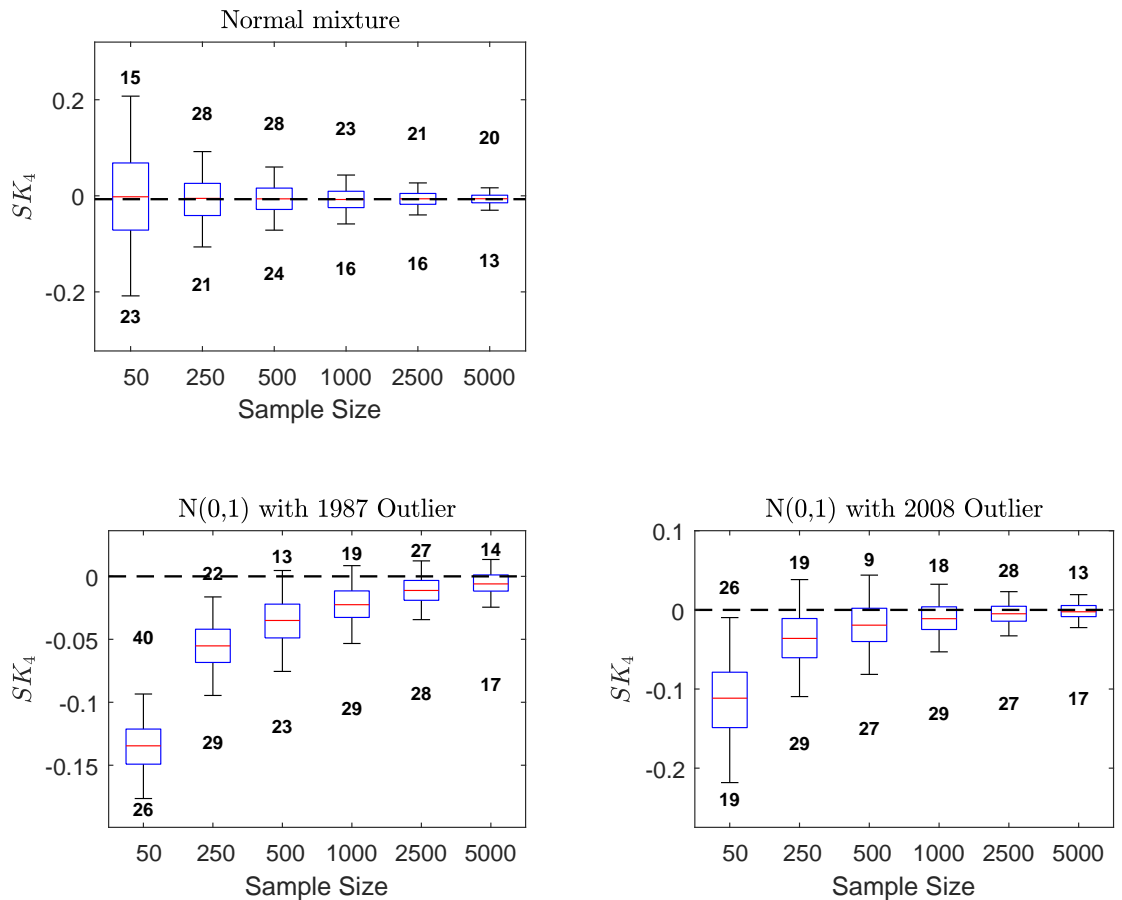

Notes: See notes to Figure 1 and details in Section 3.1.1.

Figure A16: Sample distribution of $S K_{5}$
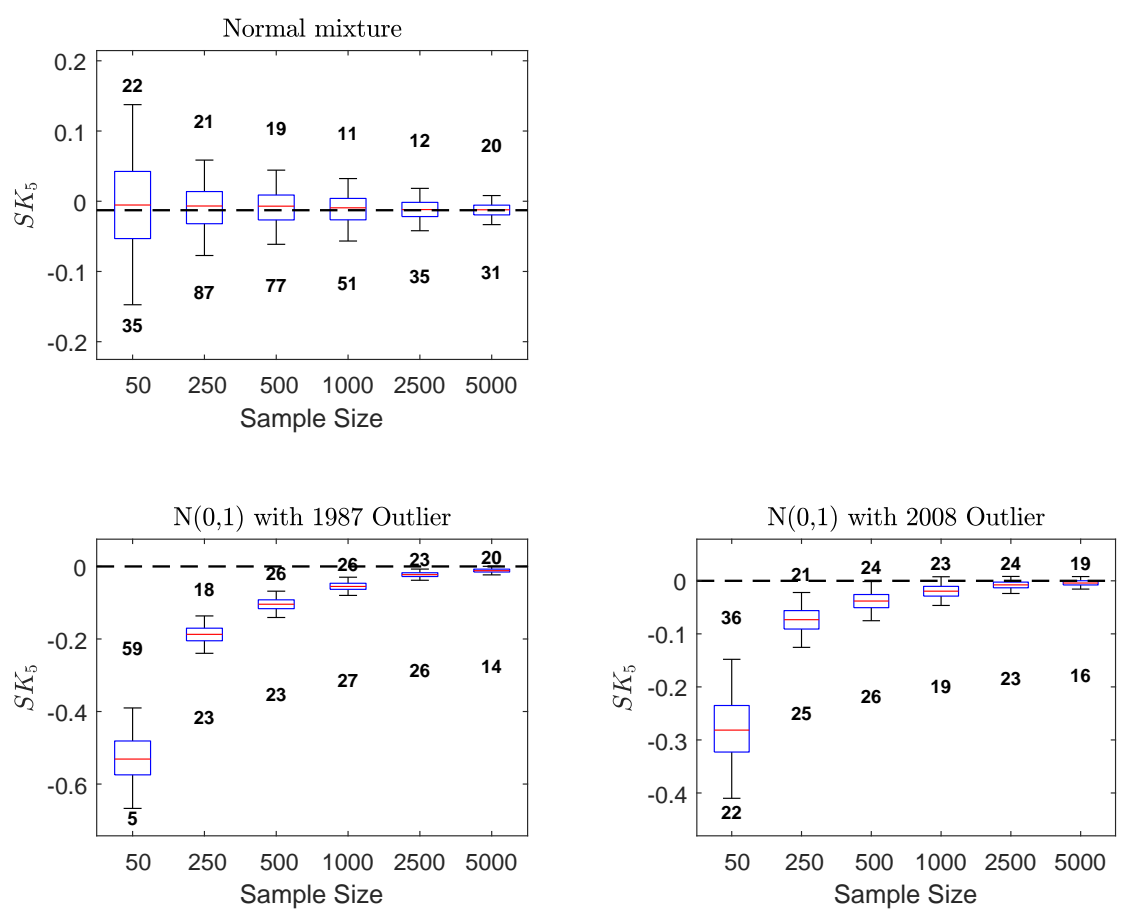

Notes: See notes to Figure 1 and details in Section 3.1.1. 
Figure A17: Sample distribution of $K R_{2}$
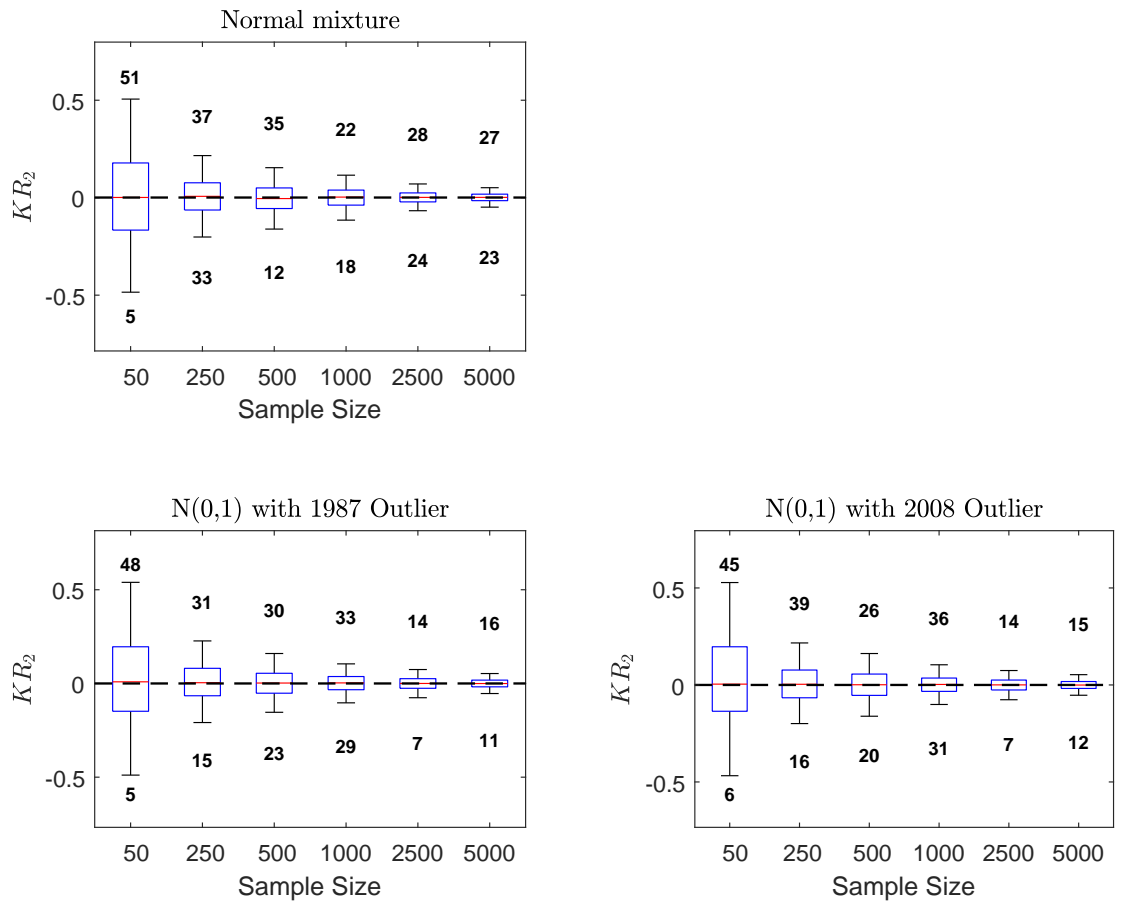

Notes: See notes to Figure 1 and details in Section 3.1.1.

Figure A18: Sample distribution of $K R_{3}$
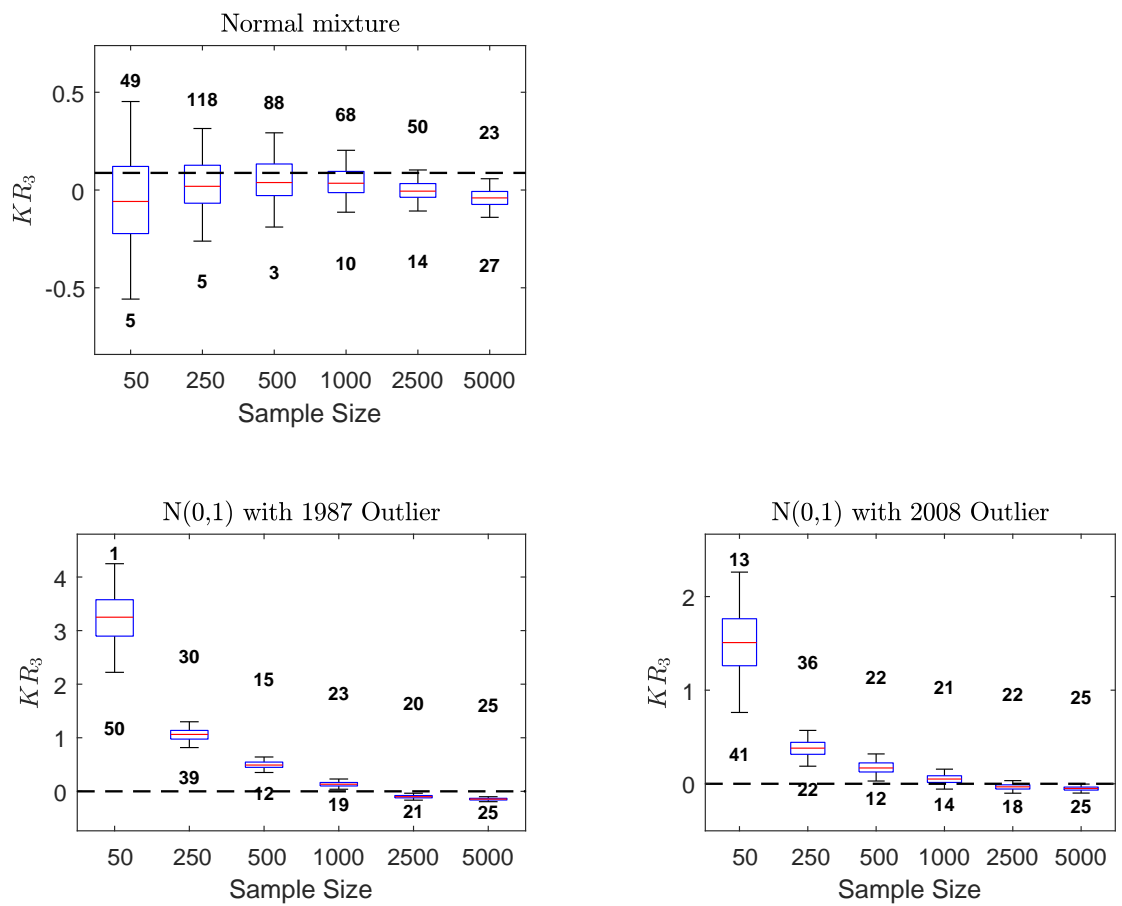

Notes: See notes to Figure 1 and details in Section 3.1.1. 
Figure A19: Sample distribution of $K R_{4}$
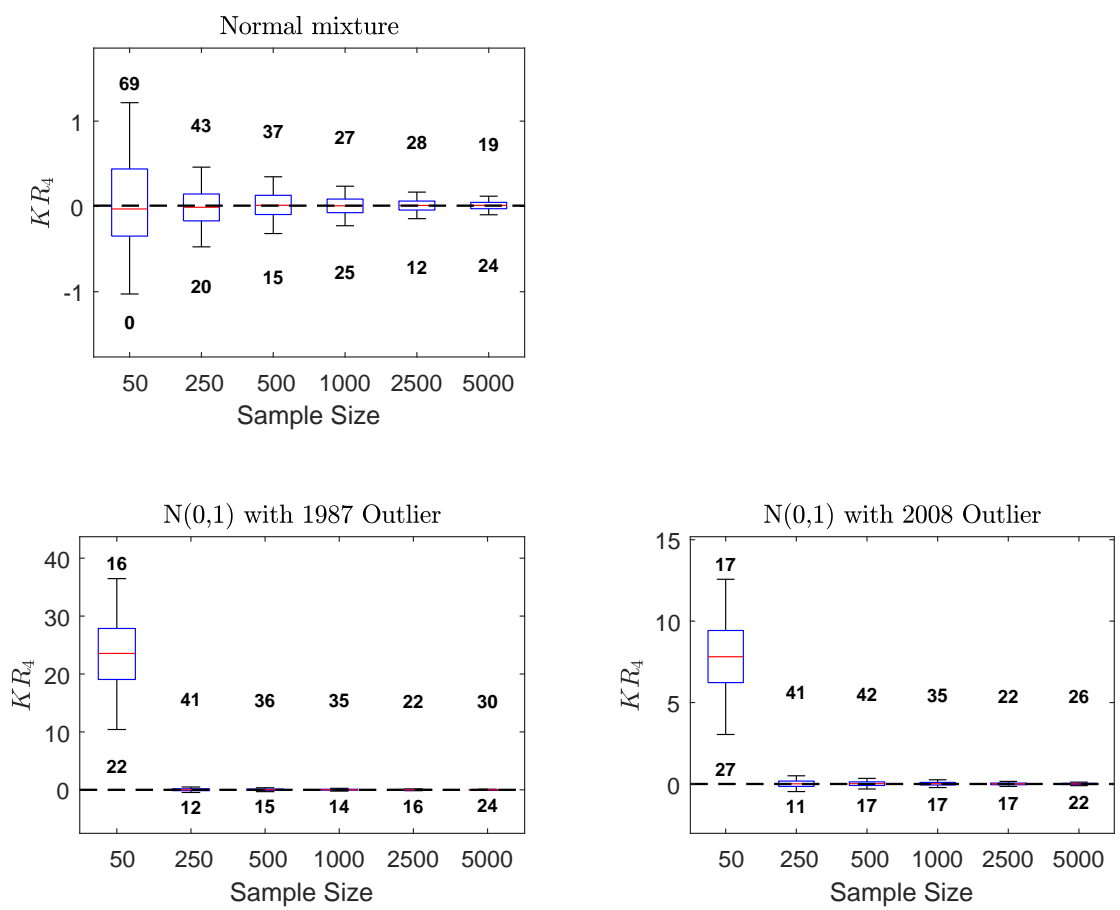

Notes: See notes to Figure 1 and details in Section 3.1.1.

Figure A20: Sample distribution of $K R_{5}$
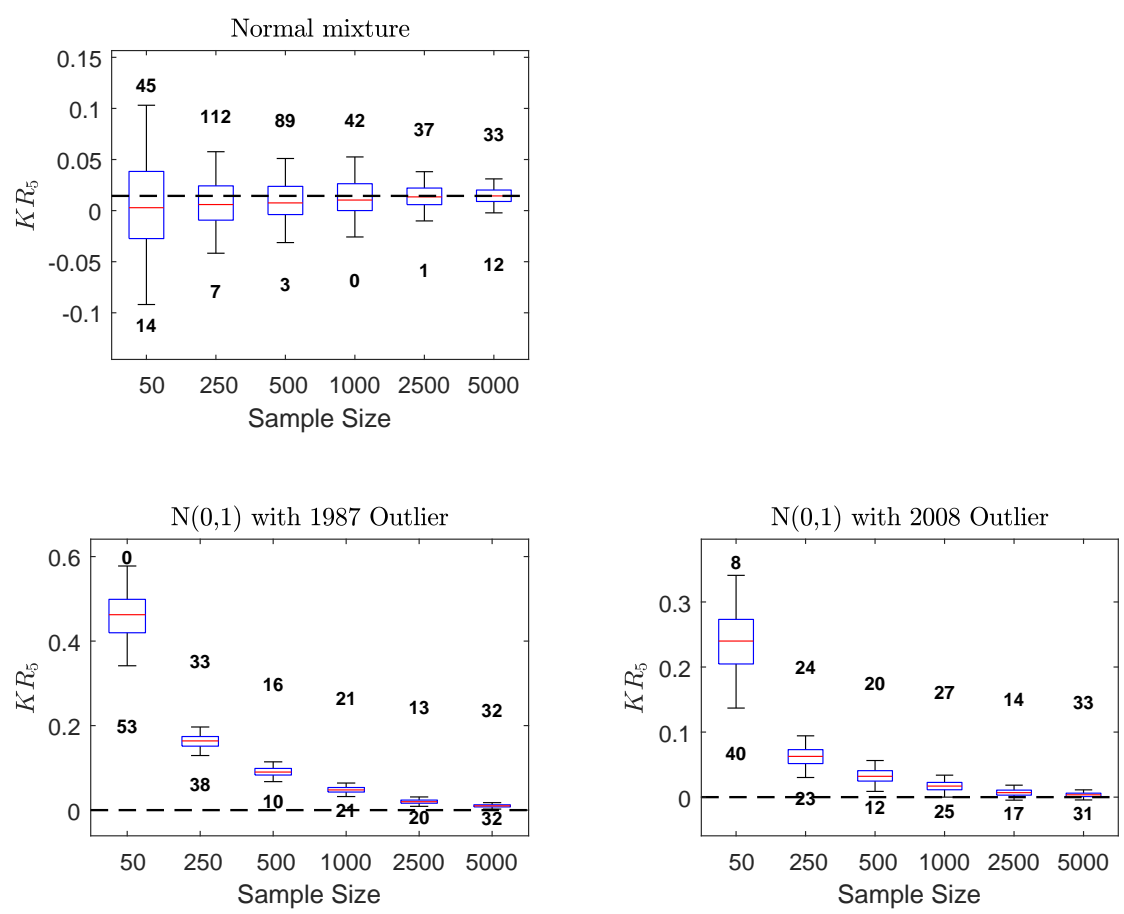

Notes: See notes to Figure 1 and details in Section 3.1.1. 


\section{D.4 Additional tables - Serial Correlation}

Table D1: Bias and RMSE ratio of skewness estimators - AR(1) model $(\rho=0.5)$

\begin{tabular}{lcccccc}
\hline$T$ & 50 & 250 & 500 & 1000 & 2500 & 5000 \\
\hline \multicolumn{7}{c}{$(a) \mathrm{N}(0,1)$} \\
\hline$S K_{1}$ & $0.010(1.000)$ & $0.001(1.000)$ & $0.004(1.000)$ & $0.003(1.000)$ & $0.001(1.000)$ & $-0.000(1.000)$ \\
$S K_{2}$ & $0.003(0.580)$ & $0.002(0.505)$ & $-0.001(0.501)$ & $-0.002(0.490)$ & $-0.000(0.513)$ & $0.000(0.539)$ \\
$S K_{3}$ & $0.004(0.420)$ & $0.000(0.374)$ & $-0.000(0.372)$ & $-0.001(0.348)$ & $0.000(0.369)$ & $-0.000(0.381)$ \\
$S K_{4}$ & $0.003(0.336)$ & $0.000(0.299)$ & $-0.000(0.297)$ & $-0.001(0.278)$ & $0.000(0.295)$ & $-0.000(0.304)$ \\
$S K_{5}$ & $0.002(0.204)$ & $0.000(0.183)$ & $0.000(0.179)$ & $0.000(0.172)$ & $0.000(0.175)$ & $-0.000(0.175)$ \\
$S K_{6}$ & $0.002(0.191)$ & $0.000(0.159)$ & $-0.000(0.155)$ & $-0.000(0.147)$ & $0.000(0.152)$ & $0.000(0.155)$ \\
\hline \multicolumn{7}{c}{$(c) t_{5}$} \\
\hline$S K_{1}$ & $0.007(1.000)$ & $0.002(1.000)$ & $-0.007(1.000)$ & $0.019(1.000)$ & $-0.009(1.000)$ & $-0.000(1.000)$ \\
$S K_{2}$ & $-0.017(0.290)$ & $0.005(0.155)$ & $-0.000(0.129)$ & $0.001(0.094)$ & $0.001(0.074)$ & $-0.001(0.081)$ \\
$S K_{3}$ & $-0.011(0.224)$ & $0.003(0.121)$ & $-0.002(0.105)$ & $0.002(0.077)$ & $-0.000(0.059)$ & $-0.000(0.065)$ \\
$S K_{4}$ & $-0.009(0.170)$ & $0.002(0.092)$ & $-0.001(0.079)$ & $0.001(0.058)$ & $-0.000(0.045)$ & $-0.000(0.049)$ \\
$S K_{5}$ & $-0.001(0.139)$ & $0.001(0.080)$ & $-0.001(0.072)$ & $0.001(0.051)$ & $-0.001(0.041)$ & $0.000(0.044)$ \\
$S K_{6}$ & $-0.003(0.094)$ & $0.001(0.055)$ & $-0.001(0.046)$ & $0.001(0.033)$ & $-0.000(0.026)$ & $-0.000(0.029)$ \\
\hline \multicolumn{7}{c}{$(d) \log -\mathrm{N}(1,0.4)$} \\
\hline$S K_{1}$ & $-0.568(1.000)$ & $-0.385(1.000)$ & $-0.371(1.000)$ & $-0.357(1.000)$ & $-0.349(1.000)$ & $-0.343(1.000)$ \\
$S K_{2}$ & $-0.041(0.257)$ & $-0.034(0.182)$ & $-0.039(0.159)$ & $-0.037(0.141)$ & $-0.037(0.124)$ & $-0.036(0.116)$ \\
$S K_{3}$ & $-0.073(0.211)$ & $-0.063(0.181)$ & $-0.066(0.175)$ & $-0.065(0.182)$ & $-0.065(0.184)$ & $-0.064(0.186)$ \\
$S K_{4}$ & $-0.052(0.158)$ & $-0.044(0.130)$ & $-0.046(0.124)$ & $-0.045(0.128)$ & $-0.045(0.128)$ & $-0.045(0.129)$ \\
$S K_{5}$ & $-0.056(0.128)$ & $-0.049(0.121)$ & $-0.050(0.125)$ & $-0.050(0.133)$ & $-0.050(0.137)$ & $-0.050(0.142)$ \\
$S K_{6}$ & $-0.045(0.106)$ & $-0.033(0.087)$ & $-0.033(0.085)$ & $-0.033(0.089)$ & $-0.032(0.090)$ & $-0.032(0.092)$ \\
\hline
\end{tabular}

Notes: see notes to Table 2. 
Table D2: Bias and RMSE ratio of excess kurtosis estimators - AR(1) model $(\rho=0.5)$

\begin{tabular}{lcccccc}
\hline$T$ & 50 & 250 & 500 & 1000 & 2500 & 5000 \\
\hline$K R_{1}$ & $-0.194(1.000)$ & $-0.030(1.000)$ & $-0.020(1.000)$ & $-0.010(1.000)$ & $-0.004(1.000)$ & $-0.002(1.000)$ \\
$K R_{2}$ & $0.013(0.438)$ & $0.006(0.378)$ & $-0.004(0.359)$ & $-0.003(0.337)$ & $0.001(0.349)$ & $0.001(0.348)$ \\
$K R_{3}$ & $-0.097(0.431)$ & $-0.015(0.381)$ & $-0.009(0.359)$ & $-0.006(0.357)$ & $-0.007(0.350)$ & $-0.009(0.370)$ \\
$K R_{4}$ & $-0.010(0.967)$ & $-0.007(0.848)$ & $0.002(0.783)$ & $0.000(0.759)$ & $0.002(0.738)$ & $0.001(0.754)$ \\
$K R_{5}$ & $-0.004(0.078)$ & $0.000(0.066)$ & $-0.001(0.062)$ & $-0.000(0.060)$ & $0.000(0.058)$ & $0.000(0.058)$ \\
$K R_{6}$ & $-0.149(0.259)$ & $-0.010(0.062)$ & $-0.003(0.053)$ & $-0.001(0.048)$ & $0.000(0.047)$ & $0.000(0.047)$ \\
\hline \multicolumn{7}{c}{$(c) t_{5}$} \\
\hline$K R_{1}$ & $-5.189(1.000)$ & $-4.155(1.000)$ & $-3.756(1.000)$ & $-3.300(1.000)$ & $-3.064(1.000)$ & $-3.075(1.000)$ \\
$K R_{2}$ & $-0.017(0.051)$ & $-0.031(0.025)$ & $-0.030(0.020)$ & $-0.033(0.012)$ & $-0.036(0.009)$ & $-0.035(0.011)$ \\
$K R_{3}$ & $-0.317(0.084)$ & $-0.177(0.048)$ & $-0.160(0.045)$ & $-0.167(0.033)$ & $-0.180(0.033)$ & $-0.192(0.048)$ \\
$K R_{4}$ & $-0.180(0.165)$ & $-0.230(0.081)$ & $-0.210(0.070)$ & $-0.228(0.051)$ & $-0.230(0.044)$ & $-0.230(0.060)$ \\
$K R_{5}$ & $-0.034(0.012)$ & $-0.025(0.007)$ & $-0.023(0.007)$ & $-0.023(0.005)$ & $-0.023(0.004)$ & $-0.022(0.006)$ \\
$K R_{6}$ & $-0.171(0.031)$ & $-0.023(0.006)$ & $-0.014(0.004)$ & $-0.011(0.003)$ & $-0.011(0.002)$ & $-0.010(0.003)$ \\
\hline \multicolumn{7}{c}{$(d) \log -\mathrm{N}(1,0.4)$} \\
\hline$K R_{1}$ & $-2.559(1.000)$ & $-1.641(1.000)$ & $-1.525(1.000)$ & $-1.435(1.000)$ & $-1.370(1.000)$ & $-1.317(1.000)$ \\
$K R_{2}$ & $-0.001(0.090)$ & $-0.012(0.050)$ & $-0.017(0.039)$ & $-0.012(0.033)$ & $-0.014(0.024)$ & $-0.013(0.020)$ \\
$K R_{3}$ & $-0.181(0.113)$ & $-0.070(0.069)$ & $-0.064(0.058)$ & $-0.064(0.054)$ & $-0.067(0.051)$ & $-0.070(0.053)$ \\
$K R_{4}$ & $-0.308(0.207)$ & $-0.150(0.137)$ & $-0.110(0.111)$ & $-0.089(0.095)$ & $-0.088(0.080)$ & $-0.081(0.071)$ \\
$K R_{5}$ & $-0.015(0.018)$ & $-0.009(0.011)$ & $-0.009(0.009)$ & $-0.009(0.008)$ & $-0.009(0.007)$ & $-0.008(0.007)$ \\
$K R_{6}$ & $-0.158(0.054)$ & $-0.015(0.010)$ & $-0.008(0.006)$ & $-0.005(0.005)$ & $-0.004(0.004)$ & $-0.004(0.004)$ \\
\hline
\end{tabular}

Notes: see notes to Table 2. 


\section{D.5 Additional tables \& figures - FRED-MD}

i

Table D3: Point estimates of maximal moment exponents

\begin{tabular}{lccc}
\hline & & $\hat{\alpha}_{\delta}$ & \\
\cline { 2 - 4 }$\delta$ & $\min$ & median & $\max$ \\
\hline $5.0 \%$ & 1.17 & 3.24 & 302.07 \\
$7.5 \%$ & 1.26 & 2.91 & 233.98 \\
$10.0 \%$ & 0.84 & 2.73 & 313.45 \\
\hline
\end{tabular}

Notes: the table shows the minimum, maximum and median value of the estimates of the maximal moment exponents $\left(\hat{\alpha}_{\delta}\right)$ for the series in the FRED-MD. The value of $\delta$ displayed in the first column of the table indicate the percentage of observations used to estimate $\hat{\alpha}_{\delta}$. Therefore $\hat{\alpha}_{10 \%}$ indicates that we rely on the upper $10 \%$ of the empirical distribution of the series. 
Figure A21: Robustness of point estimates of maximal moment exponents
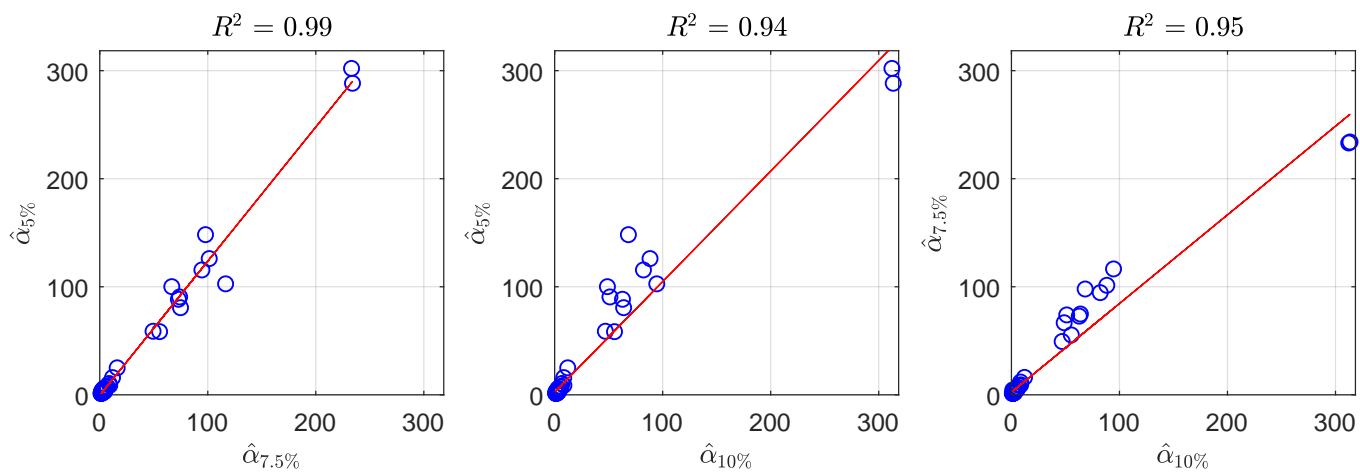

Notes: the figures report estimates of maximal moment exponents for series in the FRED-MD with different $\delta$.

Figure A22: Series in the FRED-MD with highest and lowest maximal moment exponent
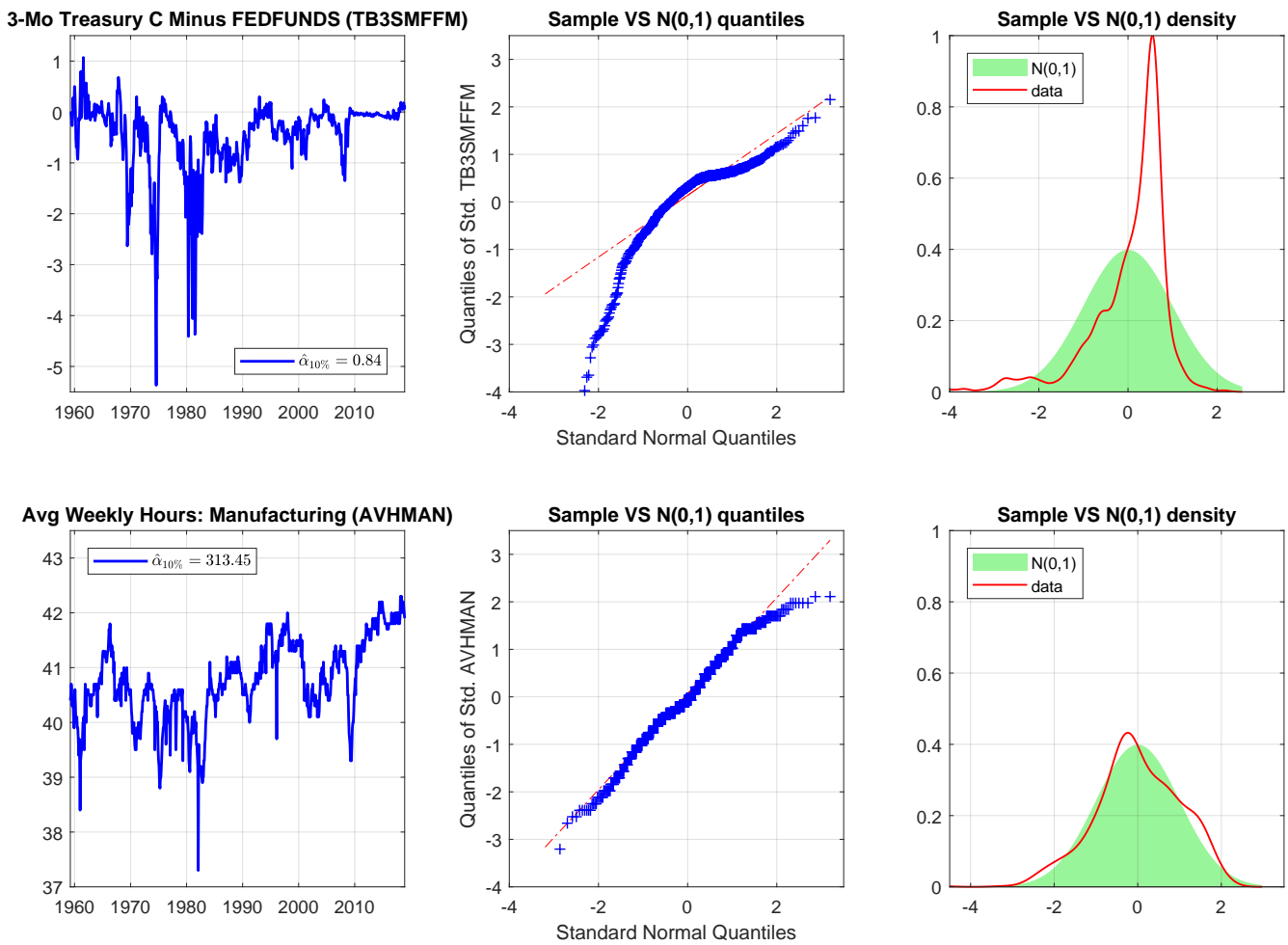